\title{
Supplemental Information
}

A multifaceted hit-finding approach reveals novel LC3-family ligands

Micah Steffek ${ }^{1 \S}$, Elizabeth Helgason ${ }^{2 \S}$, Nataliya Popovych ${ }^{2}$, Lionel Rougé ${ }^{3}$, John M. Bruning ${ }^{1}$, Ke Sherry $\mathrm{Li}^{1}$, Daniel J. Burdick ${ }^{4}$, Jianping Cai ${ }^{5}, \#$, Terry Crawford ${ }^{4}$, Jing Xue ${ }^{1}$, Willy Decurtins ${ }^{5}$, Chunlin Fang ${ }^{6}$, Felix Grubers $^{5}$, Michael J. Holliday ${ }^{2, \$}$, Allyson Langley ${ }^{2, \mathrm{c}}$, Ann Petersen ${ }^{5}$, Alexander Lee Satz ${ }^{5, *}$, Aimin Song ${ }^{2}$, Daniel Stoffler ${ }^{5}$, Quentin Strebel ${ }^{5}$, Jeffrey Y.K. Tom ${ }^{2}$, Nicholas Skelton ${ }^{4}$, Steven T. Staben ${ }^{4,}{ }^{+}$, Moreno $^{2}$ Wichert $^{5}$, Melinda M. Mulvihill ${ }^{*}$, and Erin C. Dueber ${ }^{2 *}$

${ }^{1}$ Biochemical and Cellular Pharmacology, Genentech, 1 DNA Way, South San Francisco, CA 94080

${ }^{2}$ Early Discovery Biochemistry, Genentech, 1 DNA Way, South San Francisco, CA 94080

${ }^{3}$ Structure Biology, Genentech, 1 DNA Way, South San Francisco, CA 94080

${ }^{4}$ Chemistry Departments, Genentech, 1 DNA Way, South San Francisco, CA 94080,

${ }^{5}$ Roche Pharma Research and Early Development, Roche Innovation Center, Grenzacherstrasse 124 Basel, $\mathrm{CH} 4070$

${ }^{6}$ WuXi AppTec (Wuhan) Co., Ltd., No.666 GaoXin Road, WuHan East Lake High-tech Development Zone Hubei, 430075, China,

*Email: mulvihill.melinda@gene.com and dueber.erin@gene.com

$\S M . S$. and E.H. contributed equally to this work

Present Addresses:

\#J.C.: Incyte Research Institute, Incyte Corporation, 1801 Augustine Cut-Off, Wilmington, DE 19803, United States, e-mail: jcai@incyte.com

\$M.H.: Relay Therapeutics, 399 Binney Street, $2^{\text {nd }}$ Floor, Cambridge, MA 02139, United States, e-mail: mholliday@relaytx.com

${ }^{c}$ A.L.: Moderna, 200 Technology Square, Cambridge, MA 02139, United States, e-mail: allyson.langley@modernatx.com

${ }^{¥}$ A.L.S. DNA Encoded Library Platform, WuXi AppTec, 55 Cambridge Pkwy 8th floor, Cambridge, MA 02142, United States, e-mail: alex.satz@wuxiapptec.com

+ S.T.S Lycia Therapeutics, 400 East Jamie Court, South San Francisco, CA 94080, e-mail: sstaben@lyciatx.com 
A
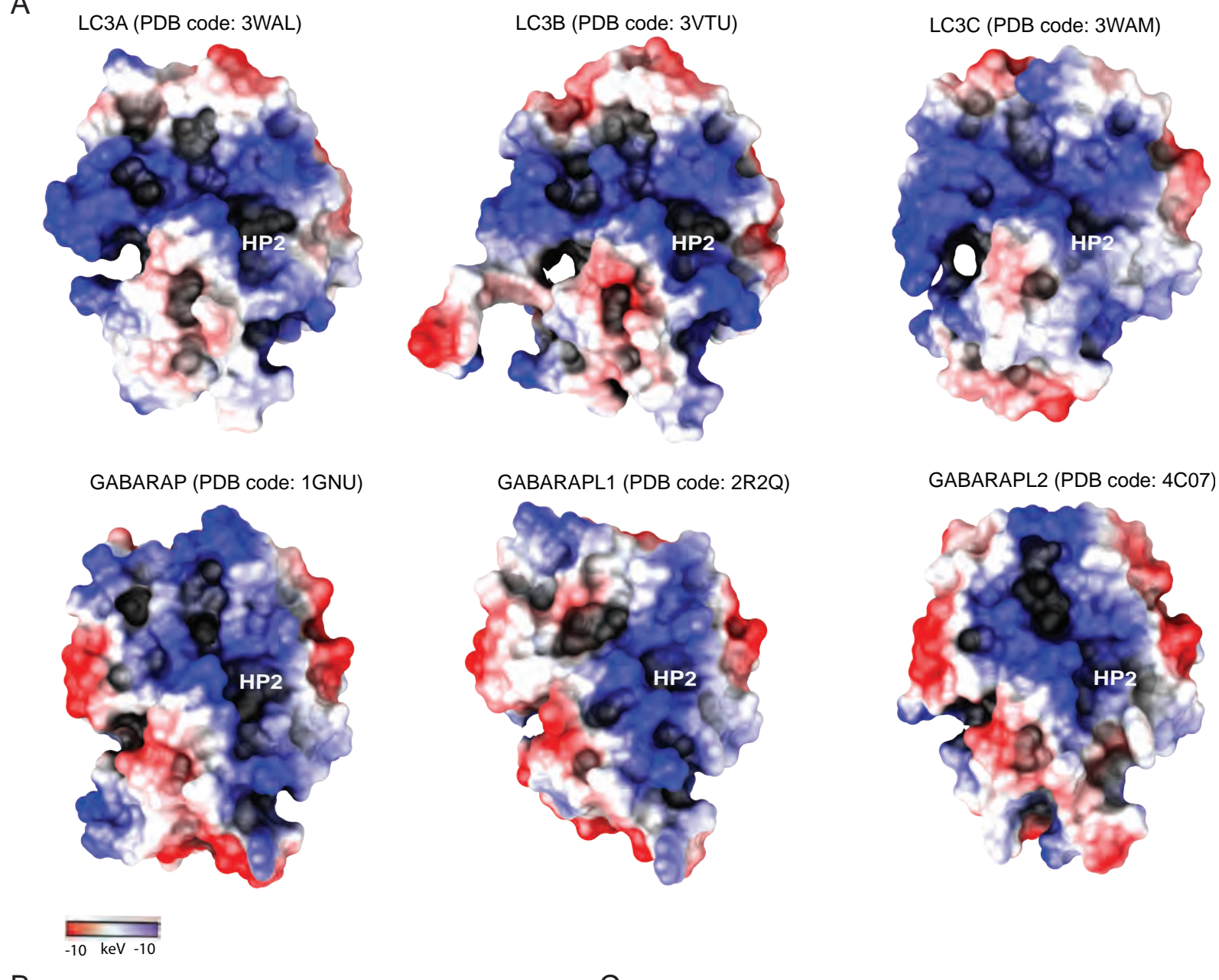

B
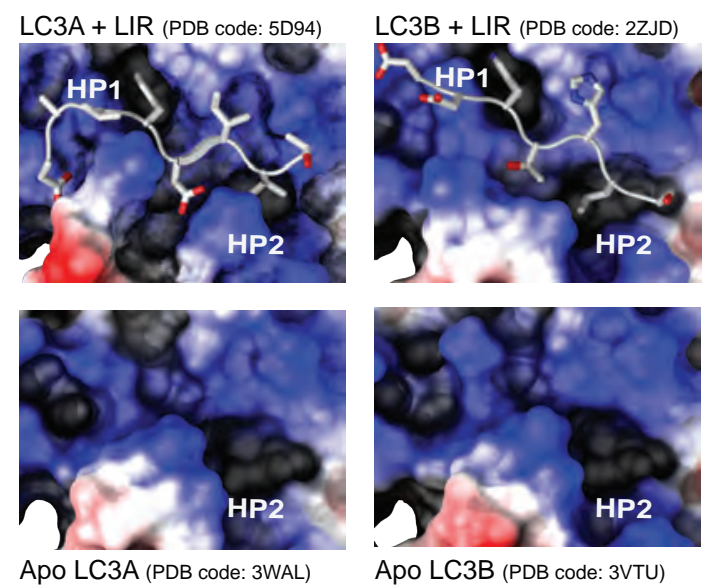

C

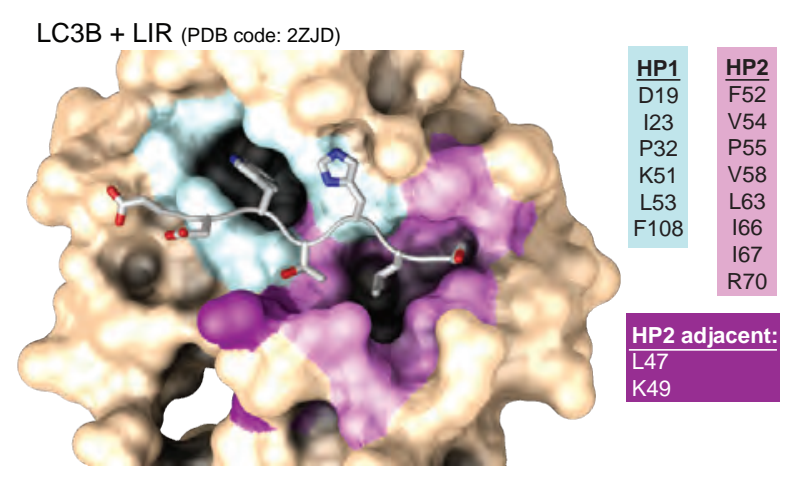

D LC3A: E19 I23 P32 K51 L53 F108 LC3B: D19 I23 P32 K51 L53 F108

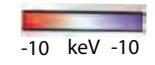

LC3A: F52 V54 P55 V58 L63 I66 I67 R70 L47 K49 LC3B: F52 V54 P55 V58 L63 I66 I67 R70

Figure S1. Comparison of mAtg8 LIR-binding sites. (A) Electrostatic charge distribution of all 6 members of the mAtg8 family. A conserved positively charged patch (blue) is observed across all proteins. Orientation is similar to Figure 2. HP2 is highlighted; however, HP1 is not fully formed in these apo structures. (B) Zoom in of LIR peptide (shown in gray sticks) bound structures for LC3A and LC3B (top left and right panels, respectively) compared to apo structures (bottom left and right panels, respectively). (C) Residues surrounding HP1 and HP2 of LC3B are highlighted. ${ }^{6,10}$ (D) LC3A and LC3B share $83 \%$ overall sequence identity with highly conserved HP1 and HP2 residues. Images were rendered using PDB codes: 3WAL, 3VTU, 3WAM, 1GNU, 2R2Q, 4C07, 5D94, and 2ZJD. 
A
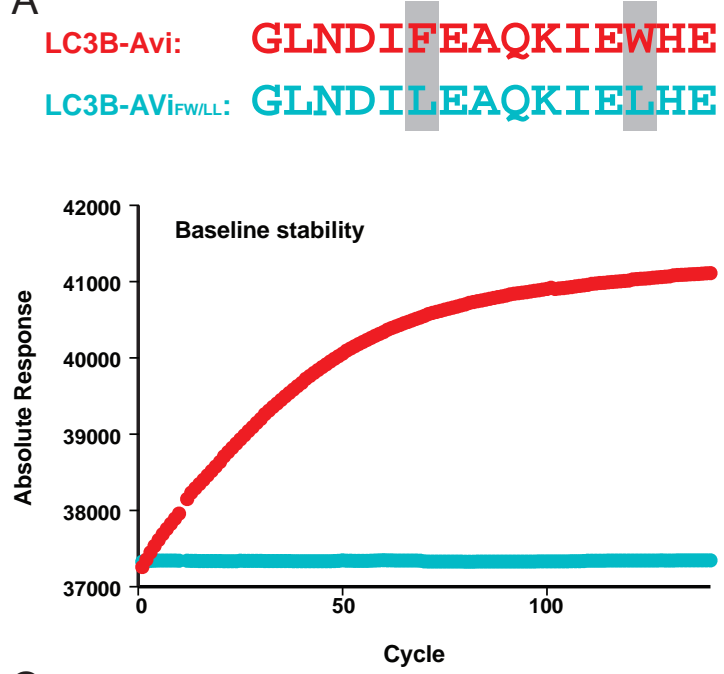

C

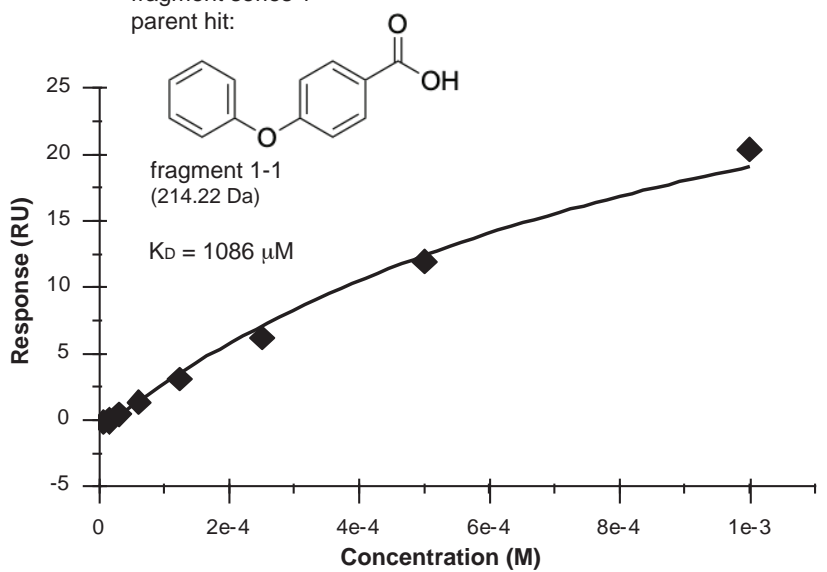

B
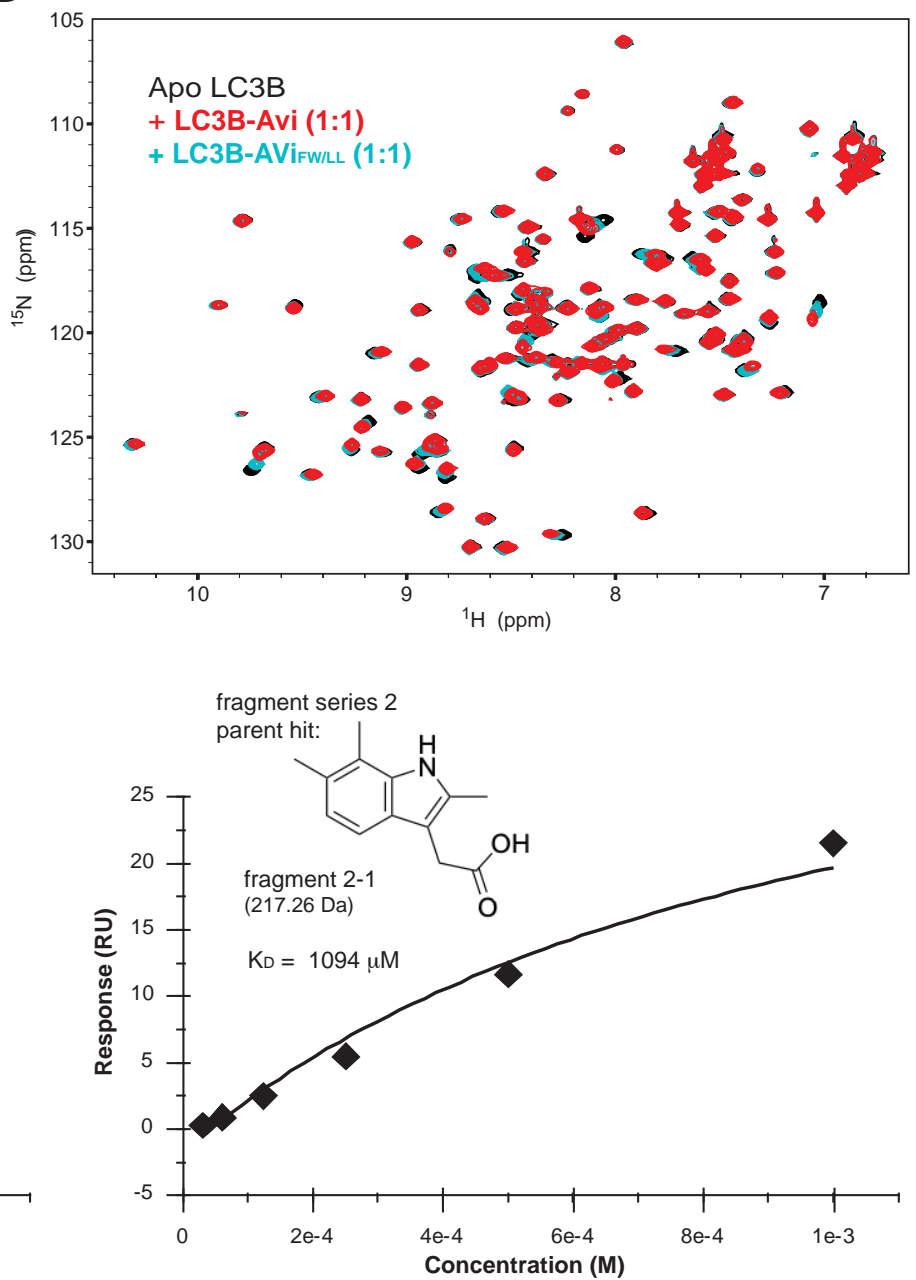

Figure S2. Improving LC3B-Avi SPR behavior for affinity measurements. (A) LC3B with the wild type Avi-tag sequence (LC3B-Avi, shown in red) and mutated Avi-tag sequence (LC3B-Avi $\mathrm{FW}_{\mathrm{LL}}$, shown in cyan) were immobilized on a SA Biacore chip and fragments were tested for binding. The absolute response for the baseline of each cycle is plotted for the duration of the binding experiment, with the increasing baseline of LC3B-Avi indicative of hydrogel collapse. (B) Overlay of ${ }^{1} \mathrm{H}-{ }^{15} \mathrm{~N}$ HSQC spectra for $200 \mu \mathrm{M}$ LC3B in the free form (black) and in the presence of either LC3B-Avi (red) or LC3B-Avi $\mathrm{FW}_{\text {/LL }}$ (cyan) in a 1:1 ratio. (C) Raw SPR data, like that shown for fragment 1-1 and fragment 2-1 in in Figure 1D, were fit using a 1:1 affinity model (left panel and right panel, respectively) to determine $K_{D}$ values. 
A

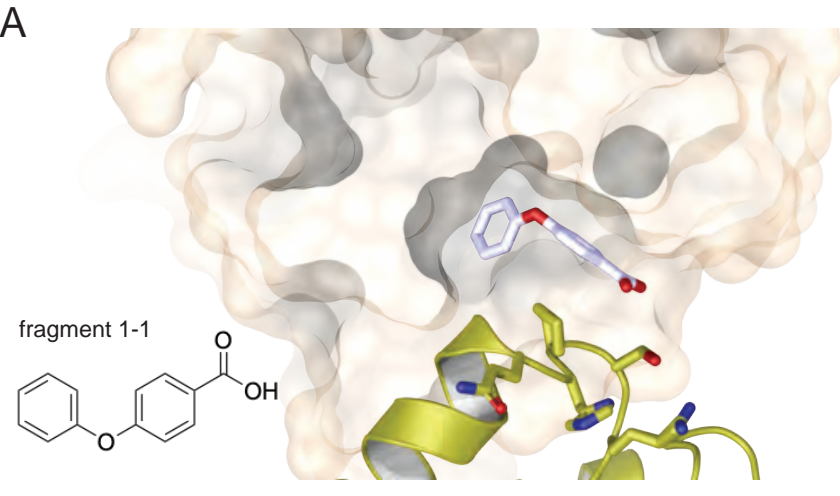

B
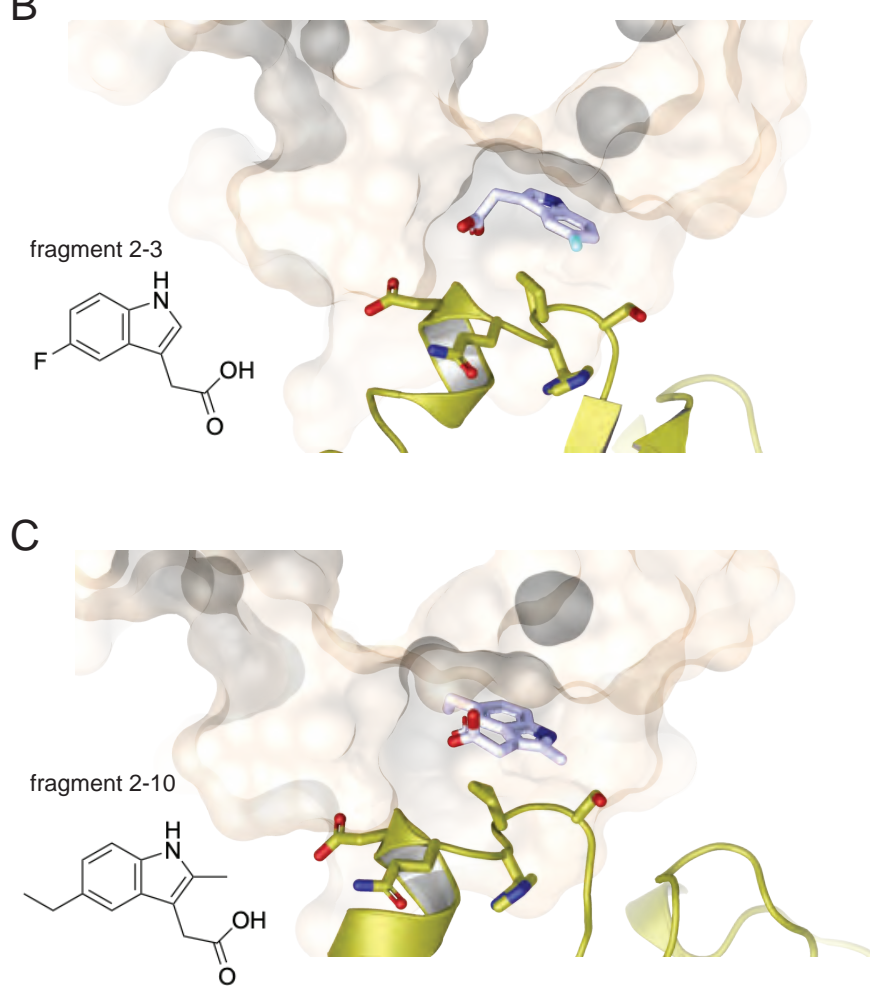

$\mathrm{D}$
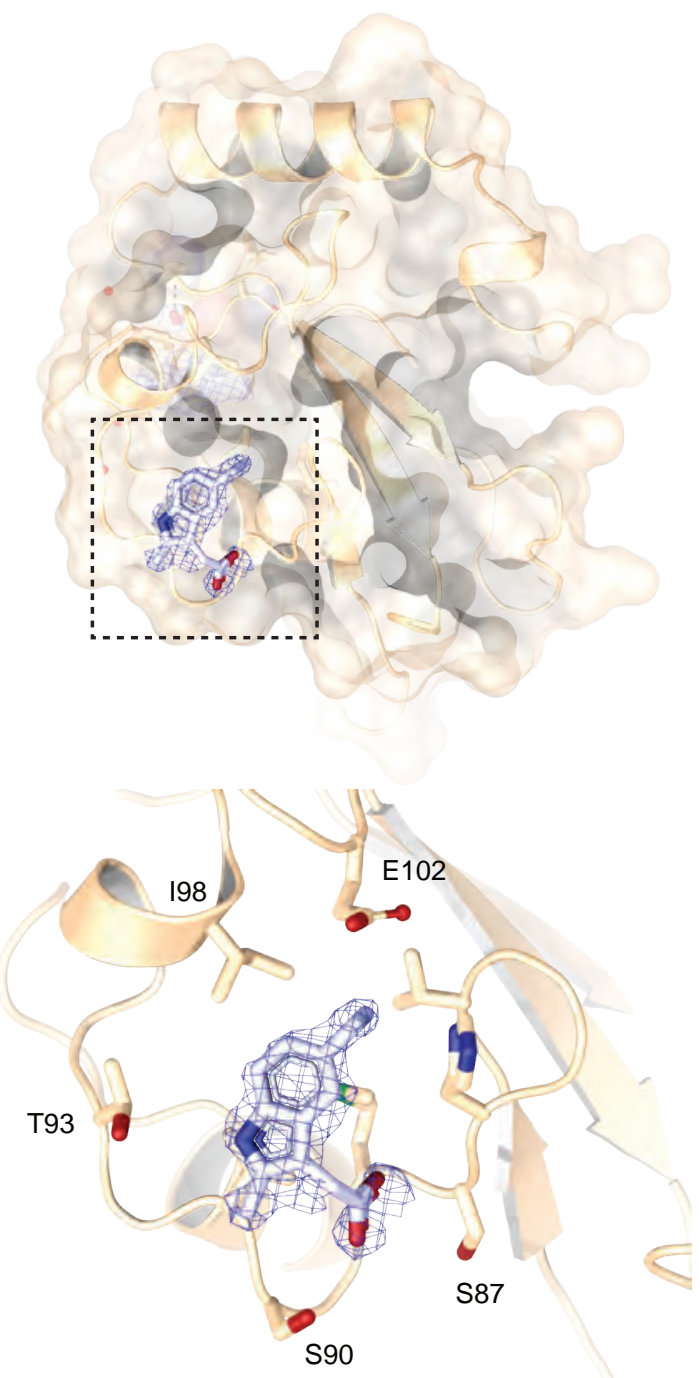

Figure S3. Overview of fragment binding sites. Crystal packing organization indicates that (A) fragment 11, (B) fragment 2-3 and (C) fragment 2-10 bind HP2 at the interface between LC3A molecules (symmetry related molecule is in light green, side chains for nearby residues and fragments are shown in sticks). While fragment 1-1 binds deeply into HP2, fragments 2-3 and 2-4 appear to make contact with the symmetry related LC3A molecule. Schematics of fragment chemical structures are shown on the left for reference. (D) Overview of fragment 2-10 second binding site. Top panel shows co-structure rotated $90^{\circ}$ compared to Figure S3C. Bottom panel shows close-up view of secondary binding site. Side chains for residues located within $5 \AA$ of the fragments are shown in sticks. 2Fo-Fc map for second fragment 2-10 molecule is shown in blue mesh contoured at $1 \sigma$. 
A

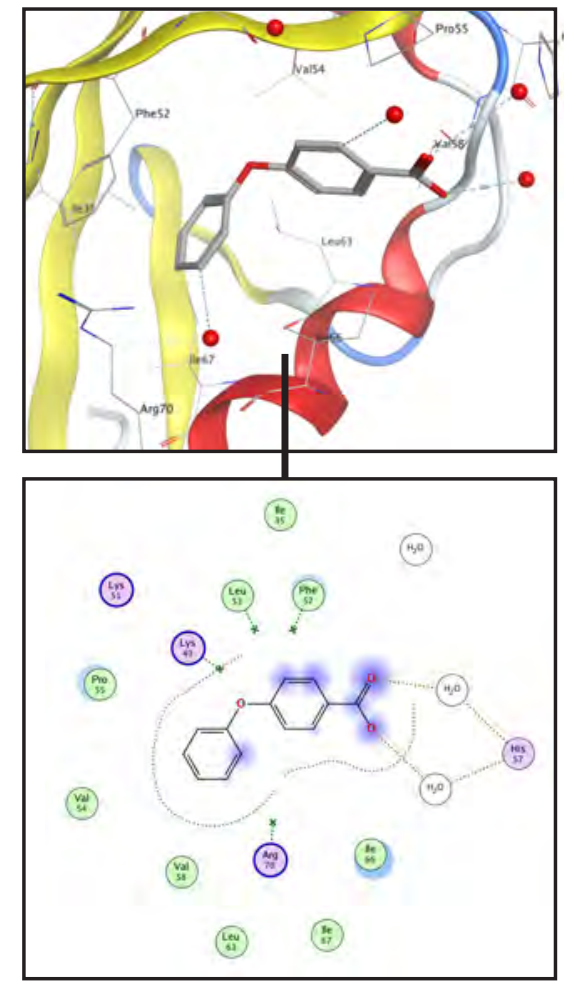

B
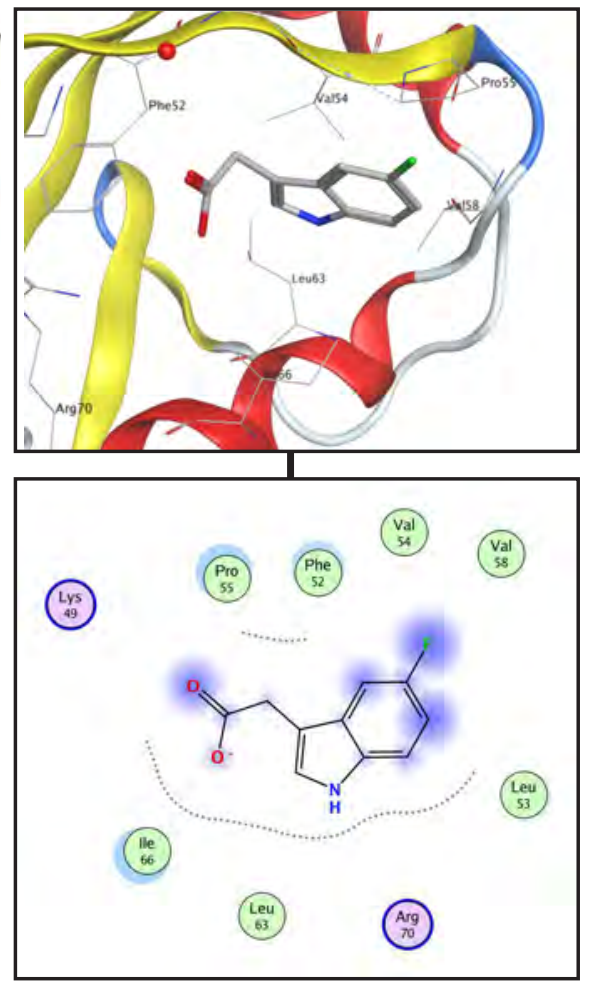

C
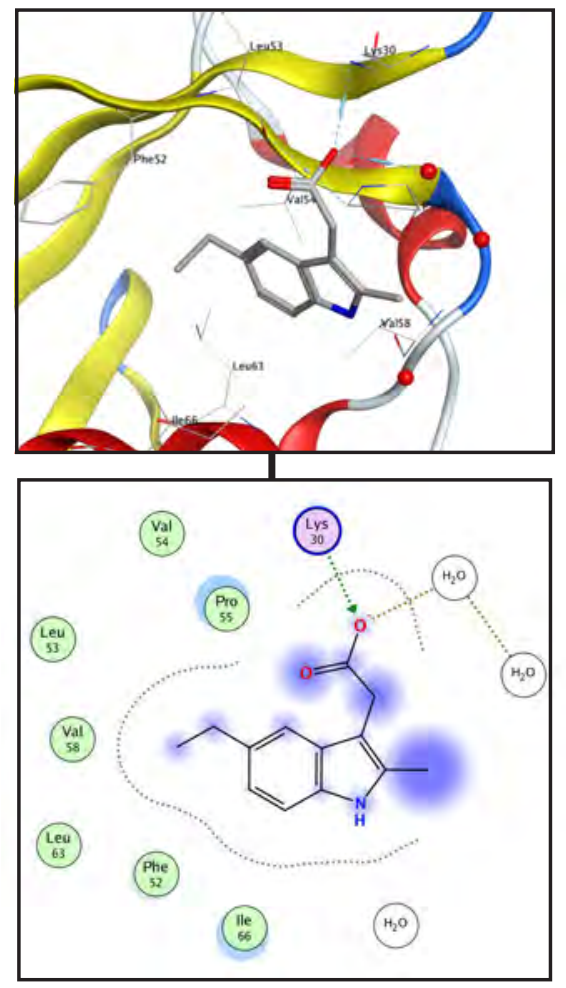

D

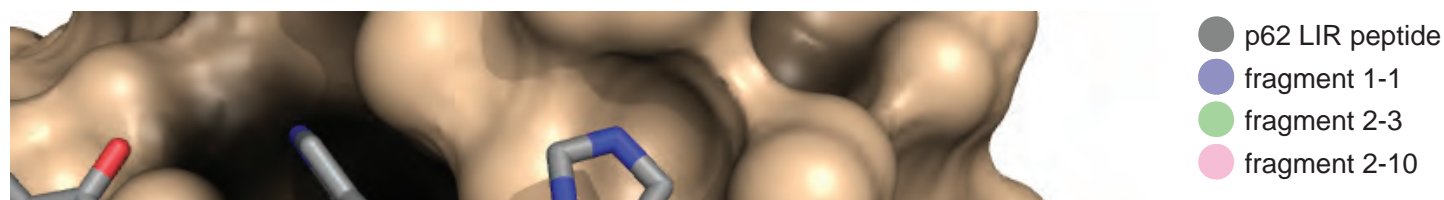

Figure S4. Structural analysis of fragment binding. Detailed views of fragment binding (top panels) and schematic interaction maps (bottom panels) for LC3A bound to (A) fragment 1-1, (B) fragment 2-3 and (C) fragment 2-10. Solvent-exposed ligand atoms are highlighted by purple spheres. Images were made using MOE software (Chemical Computing Group). (D) LIR-bound LC3B overlaid with fragments 1-1, 2-3 and 210 from LC3A co-crystal structures highlight fragment binding to HP2. Overlays created by aligning LC3A to LC3B for each of the co-crystal structures. 


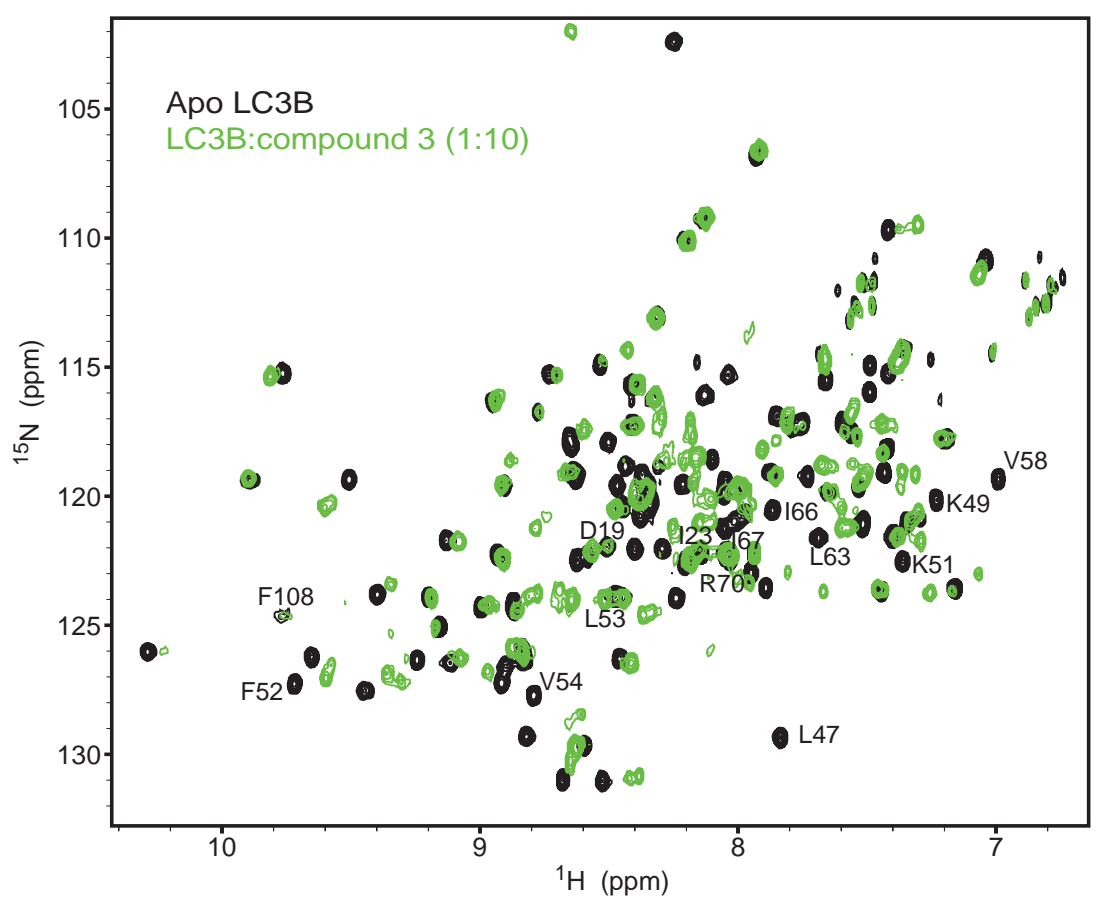

Figure S5. Compound 3 binds at or near LIR-binding site on LC3B. Overlay of ${ }^{1} \mathrm{H}-{ }^{15} \mathrm{~N}$ TROSY-HSQC spectra for $100 \mu \mathrm{M}$ LC3B in the free form (black) and in the presence of 10-fold molar excess of compound 3 (green), with HP1 and HP2 peaks labeled. Peaks corresponding to residues surrounding HP2 and K51 of HP1 are broadened or shifted due to binding. 
Table S1. Crystallographic data collection and refinement statistics (*values in parentheses are for high-resolution shell)

\begin{tabular}{|c|c|c|c|}
\hline PDB code & $\begin{array}{c}\mathrm{LC} 3 \mathrm{~A}+\text { tragment } \mathrm{I}-\mathrm{I} \\
/ \mathrm{KYW}\end{array}$ & $\begin{array}{c}\mathrm{LC} 3 \mathrm{~A}+\text { tragment } 2-3 \\
/ \mathrm{KYL}\end{array}$ & $\begin{array}{c}\mathrm{LC} 3 \mathrm{~A}+\text { tragment } 2-10 \\
\text { /RA } 0\end{array}$ \\
\hline \multicolumn{4}{|l|}{ Data Collection } \\
\hline Space Group & $14_{1}$ & $14_{1}$ & $14_{1}$ \\
\hline \multicolumn{4}{|l|}{ C'ell dimensions } \\
\hline $\mathrm{a}, \mathrm{b}, \mathrm{c}(\AA)$ & $94.02,94.02,33$ & $93.46,93.46,32.32$ & $93.62,93.62,32.84$ \\
\hline$\alpha, \beta, \gamma\left({ }^{\sim}\right)$ & $y 0, y 0, y 0$ & $y 0, y 0, y 0$ & $y 0, y 0, y 0$ \\
\hline Resolution range (A) & $23.69-1.75(1.78-1.75)$ & $46.73-1.72(1.75-1.72)$ & $33.10-1.36(1.38-1.36)$ \\
\hline $\mathrm{K}_{\text {merg }}(\%)$ & $0.038(0.528)$ & $0.052(1.250)$ & $0.057(1.217)$ \\
\hline$<\mathrm{l}>|<\sigma \mathrm{l}\rangle$ & $1 \% .5(2.4)$ & $22.9(2.0)$ & $20.1(2.2)$ \\
\hline Completeness (\%) & 99.1 (98.5) & $100(100)$ & $100(100)$ \\
\hline Redundancy & $6.1(5.9)$ & $12.9(12.1)$ & $12.8(12.5)$ \\
\hline
\end{tabular}

\section{Refinement}

Resolution $(\AA)$

No. atoms

\section{Protein}

Ligand/1on

Water

\section{0}

B-tactors ( $\mathrm{A}^{-}$)

Protein

Ligand/1on

Water

K.m.s. deviatıons

\section{Ramachandran}


Table S2. Fragment Series 1 affinities by SPR

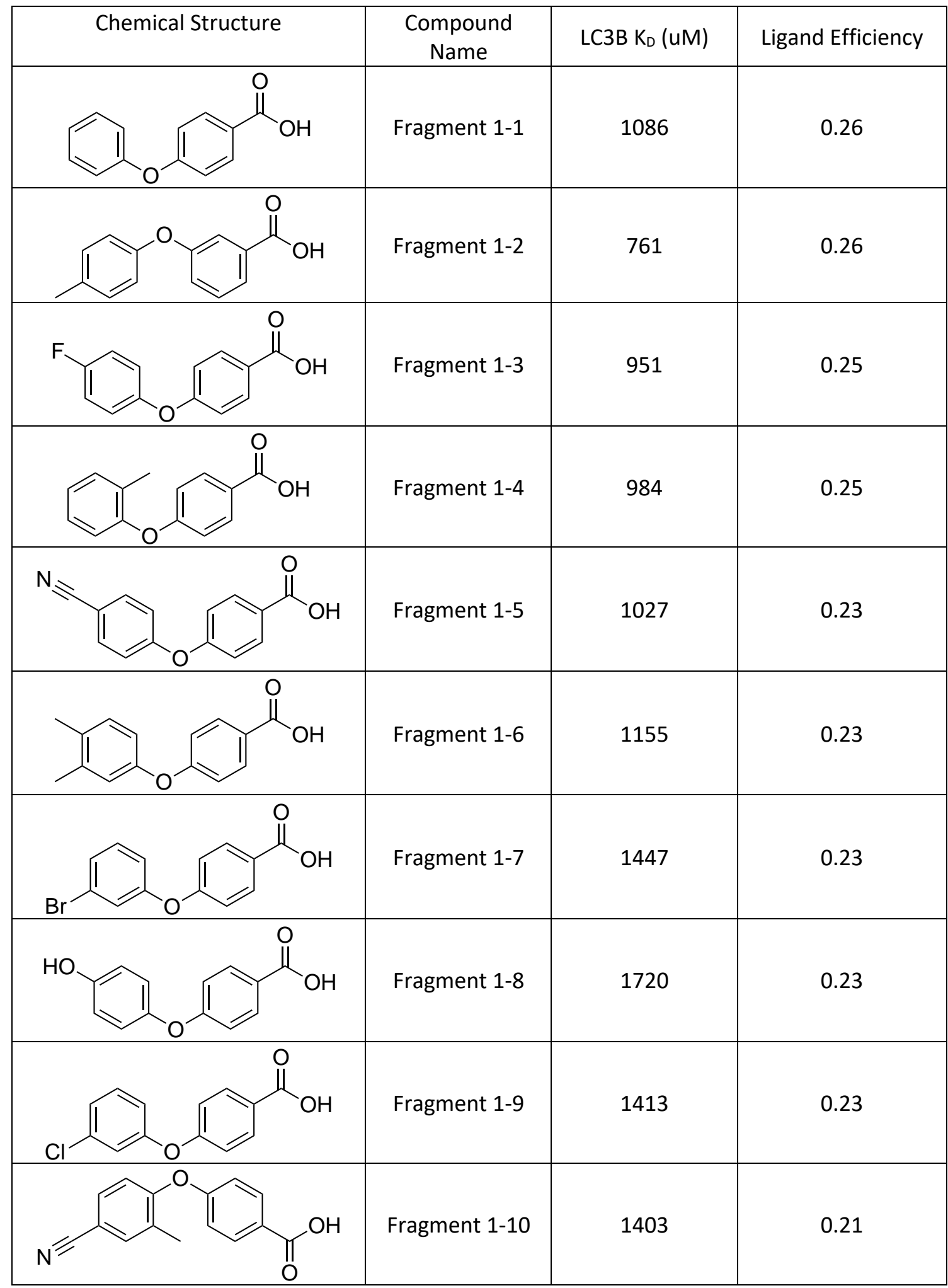




\begin{tabular}{|l|l|l|l|}
\hline & & \\
\hline Fragment 1-13 & $>3010$ \\
\hline
\end{tabular}


Table S3. Fragment Series 2 affinities by SPR

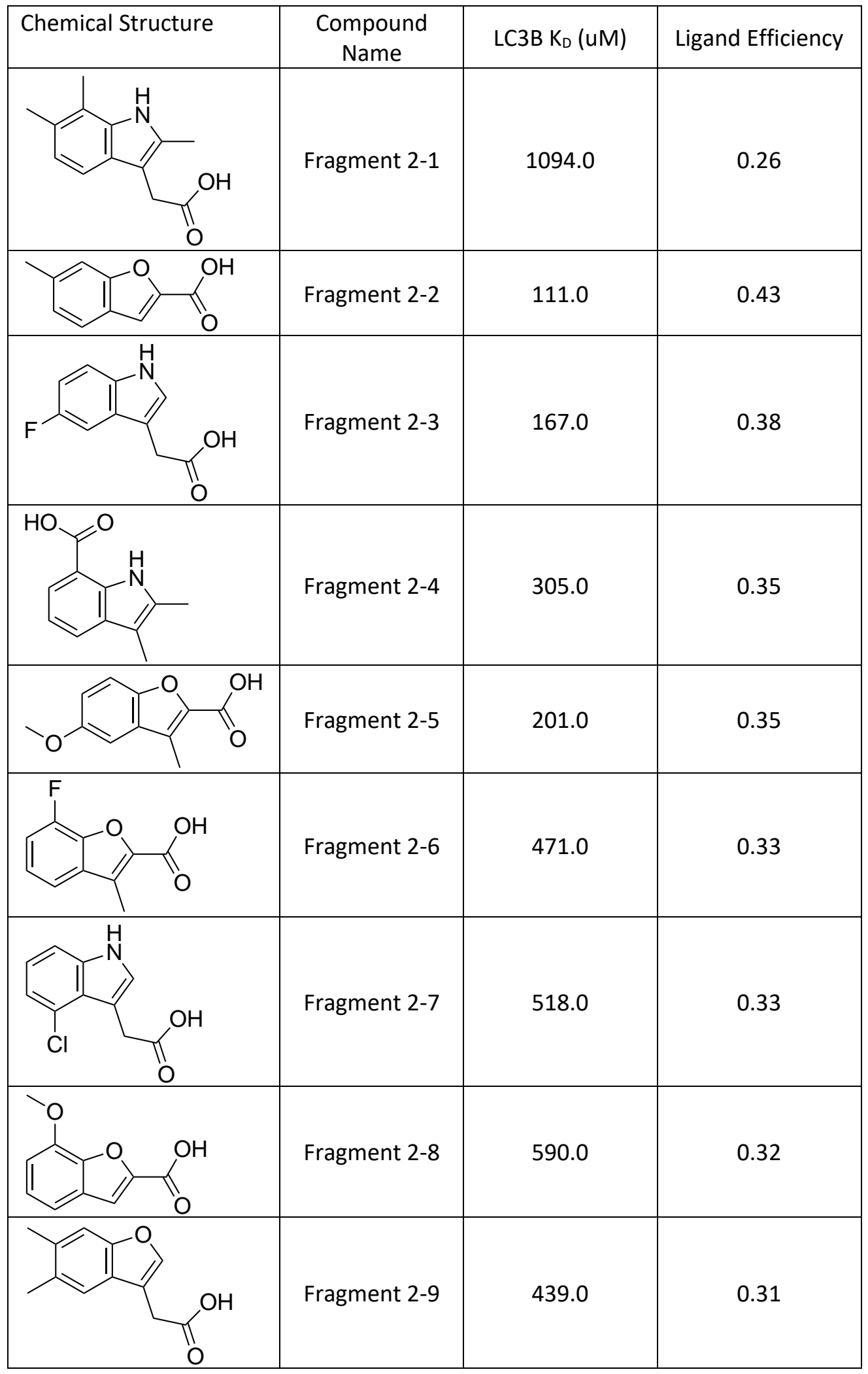




\begin{tabular}{|l|l|l|}
\hline \\
\hline
\end{tabular}




\begin{tabular}{|l|l|l|l|}
\hline & Fragment 2-18 & $>3000$ \\
\hline
\end{tabular}


Table S4. DEL compound affinities by SPR

\begin{tabular}{|c|c|c|c|c|c|c|c|}
\hline & & Steady State & & & State Mo & & \\
\hline & Compound Name & LC3B KD ( $\mu \mathrm{M})$ & LC3B KD $(\mu \mathrm{M})$ & ka1 (1/Ms) & $\mathrm{kd} 1(1 / \mathrm{s})$ & ka2 (1/s) & $\mathrm{kd} 2(1 / \mathrm{s})$ \\
\hline & compound $1^{a}$ & & 1.58 & $8.9 \mathrm{E}+02$ & $1.5 \mathrm{E}-03$ & $6.0 \mathrm{E}-05$ & $1.1 \mathrm{E}-03$ \\
\hline & compound $2^{a}$ & & 0.59 & $3.6 \mathrm{E}+04$ & $5.6 \mathrm{E}-02$ & 7.6E-03 & 4.7E-03 \\
\hline & compound $3^{b}$ & & 1.6 & $3.8 \mathrm{E}+04$ & $2.6 \mathrm{E}-01$ & $1.2 \mathrm{E}-02$ & 1.4E-02 \\
\hline & compound $4^{a}$ & & 2 & $5.8 \mathrm{E}+02$ & $8.8 \mathrm{E}-03$ & $1.8 \mathrm{E}-02$ & $1.8 \mathrm{E}-03$ \\
\hline & compound $5^{\mathrm{a}}$ & & 4.4 & $5.8 \mathrm{E}+02$ & $3.3 \mathrm{E}-02$ & $4.8 \mathrm{E}-02$ & $5.5 \mathrm{E}-03$ \\
\hline & compound $6^{\mathrm{a}}$ & 41 & & & & & \\
\hline & compound $7^{\mathrm{a}}$ & 57 & & & & & \\
\hline & compound $8^{b}$ & $>100$ & & & & & \\
\hline & compound $9^{b}$ & $>100$ & & & & & \\
\hline
\end{tabular}




\section{Experimental Procedure:}

\section{Scheme 1:}

A.<smiles>[R]c1ccc(C(CC(=O)O)n2c(-c3c([R])c([R])c([R3])c([R])c3[R])nc3cc(C(=O)NC)ccc32)cc1[R]</smiles>

B.

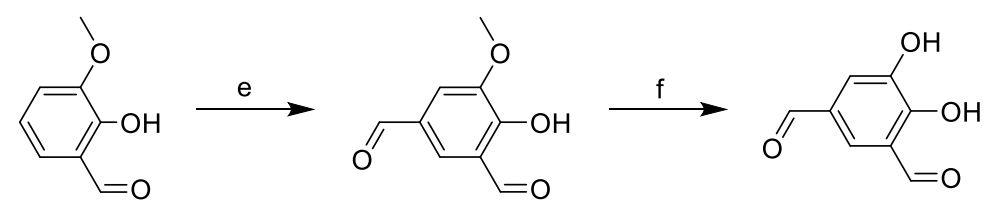

\begin{tabular}{|c|c|c|c|c|c|c|c|}
\hline Compound & $\mathrm{R} 1$ & $\mathrm{R} 2$ & $\mathrm{R} 3$ & $\mathrm{R} 4$ & $\mathrm{R} 5$ & $\mathrm{R} 6$ & $\mathrm{R} 7$ \\
\hline 1 & $\mathrm{OPh}$ & $\mathrm{H}$ & $\mathrm{OH}$ & $\mathrm{CHO}$ & $\mathrm{H}$ & $\mathrm{OEt}$ & $\mathrm{H}$ \\
\hline 2 & $\mathrm{OPh}$ & $\mathrm{H}$ & $\mathrm{OH}$ & $\mathrm{OH}$ & $\mathrm{H}$ & $\mathrm{CHO}$ & $\mathrm{H}$ \\
\hline 3 & $\mathrm{OPh}$ & $\mathrm{H}$ & $\mathrm{H}$ & $\mathrm{CHO}$ & $\mathrm{OH}$ & $\mathrm{OH}$ & $\mathrm{H}$ \\
\hline 4 & $\mathrm{H}$ & $\mathrm{OMe}$ & $\mathrm{OH}$ & $\mathrm{CHO}$ & $\mathrm{H}$ & $\mathrm{OEt}$ & $\mathrm{H}$ \\
\hline 5 & $\mathrm{H}$ & $\mathrm{F}$ & $\mathrm{OH}$ & $\mathrm{CHO}$ & $\mathrm{H}$ & $\mathrm{OEt}$ & $\mathrm{H}$ \\
\hline 6 & $\mathrm{OPh}$ & $\mathrm{H}$ & $\mathrm{OH}$ & $\mathrm{H}$ & $\mathrm{Cl}$ & $\mathrm{H}$ & $\mathrm{Cl}$ \\
\hline 7 & $\mathrm{OPh}$ & $\mathrm{H}$ & $\mathrm{H}$ & $\mathrm{CHO}$ & $\mathrm{OH}$ & $\mathrm{H}$ & $\mathrm{H}$ \\
\hline 8 & $\mathrm{H}$ & $\mathrm{OMe}$ & $\mathrm{OH}$ & $\mathrm{COOH}$ & $\mathrm{H}$ & $\mathrm{OEt}$ & $\mathrm{H}$ \\
\hline 9 & $\mathrm{H}$ & $\mathrm{F}$ & $\mathrm{OH}$ & $\mathrm{COOH}$ & $\mathrm{H}$ & $\mathrm{OEt}$ & $\mathrm{H}$ \\
\hline
\end{tabular}

(a) $(\mathrm{COCl})_{2}, \mathrm{DCM} ; \mathrm{MeNH}_{2}, \mathrm{DMF}$; (b) appropriate beta-phenylglycine, $\mathrm{NaHCO}_{3}, \mathrm{EtOH} / \mathrm{H}_{2} \mathrm{O}, 90{ }^{\circ} \mathrm{C}, 12 \mathrm{~h}$; (c) $\mathrm{Pd} / \mathrm{C}, \mathrm{MeOH}, 20^{\circ} \mathrm{C}, 6 \mathrm{~h}, \mathrm{H}_{2}$ (15 psi); (d) 1.5 eq 6, DMF, $60{ }^{\circ} \mathrm{C}, 12 \mathrm{~h}$; (e) hexamethylenetetramine (3 eq.), TFA, $90^{\circ} \mathrm{C}, 24 \mathrm{~h}$; (f) $\mathrm{HBr}, 130^{\circ} \mathrm{C}, 3 \mathrm{~h}$

Compounds were prepared as shown in Scheme 1A. 4-fluoro-N-methyl-3-nitrobenzamide was generated in good yield from 4-fluoro-3-nitrobenzoic acid using oxalyl chloride followed by methylamine. Reduction of the nitro group followed by $S_{N} A R$ with the appropriate beta-phenylglycine afforded the di-aniline which was then cyclized with the appropriate aldehyde to give final compounds 1 and 4-9. 2-hydroxy-3methoxybenzaldehyde was treated with hexamethylenetetramine followed by de-methylation with $\mathrm{HBr}$ to generate the di-aldehyde (Scheme 1B). The di-aldehyde was condensed with the appropriate di-aniline to give a mixture of products which were separated by prep HPLC to afford compounds 2 and 3 . All final compounds were analyzed by LCMS and proton NMR to verify purity and identity.

\section{4-fluoro-N-methyl-3-nitro-benzamide (2)}<smiles>O=C(O)c1ccc(F)c([N+](=O)[O-])c1</smiles>

1<smiles>CC(C)(C)OC(=O)CCNC(=O)c1ccc(F)c([N+](=O)[O-])c1</smiles>

2 
To a solution of 4-fluoro-3-nitrobenzoic acid $(10.0 \mathrm{~g}, 54.02 \mathrm{mmol})$ and N,N-dimethylformamide (789.69 $\mathrm{mg}, 10.8 \mathrm{mmol})$ in $\mathrm{DCM}(100 \mathrm{~mL})$ was added ethanedioyl dichloride $(20.57 \mathrm{~g}, 162.07 \mathrm{mmol})$ at $0{ }^{\circ} \mathrm{C}$, the reaction was stirred for $1 \mathrm{~h}$ at $25^{\circ} \mathrm{C}$, then the reaction mixture was concentrated. The residue was dissolved in DCM (50 mL) and then drop into a stirring mixture of $\mathrm{N}, \mathrm{N}$-diisopropylethylamine (20.95 $\mathrm{g}$, $162.07 \mathrm{mmol}$ ) and methanamine hydrochloride $(4.01 \mathrm{~g}, 59.42 \mathrm{mmol})$ in DCM $(100 \mathrm{~mL})$, the reaction was stirred for $1 \mathrm{~h}$ at $25{ }^{\circ} \mathrm{C}$. LCMS (5-95AB/1.5min): RT $=0.732 \mathrm{~min},[\mathrm{M}+\mathrm{H}]^{+} 199.1$ showed $96 \%$ of DP. The reaction mixture was washed with $1 \mathrm{M} \mathrm{HCl}$ aqueous $(100 \mathrm{~mL})$ and then washed with brine $(100 \mathrm{~mL})$, the organic phase was dried by $\mathrm{Na}_{2} \mathrm{SO}_{4}$, concentrated, and the residue was washed with MTBE $(30 \mathrm{~mL})$ to give 4-fluoro-N-methyl-3-nitro-benzamide ( $8.5 \mathrm{~g}, 42.897 \mathrm{mmol}, 79.4 \%$ yield) as yellow solid confirmed by ${ }^{1} \mathrm{H}$ NMR (400 MHz, CHLOROFORM-d) $\delta=8.54-8.46(\mathrm{~m}, 1 \mathrm{H}), 8.20-8.10(\mathrm{~m}, 1 \mathrm{H}), 7.38(\mathrm{dd}, \mathrm{J}=8.7,10.2 \mathrm{~Hz}$, 1H), $3.05(\mathrm{~d}, \mathrm{~J}=4.8 \mathrm{~Hz}, 3 \mathrm{H})$

\section{Compound 1:}

\section{3-[4-(methylcarbamoyl)-2-nitro-anilino]-3-(3-phenoxyphenyl)propanoic acid (3)}

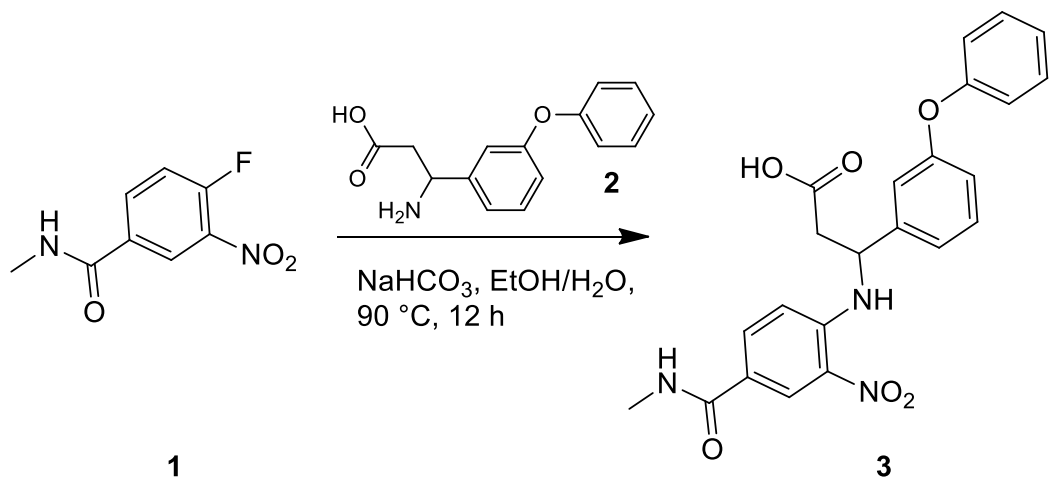

To a solution of 4-fluoro- $\mathrm{N}$-methyl-3-nitro-benzamide $(1.6 \mathrm{~g}, 8.07 \mathrm{mmol})$ in ethanol $(15 \mathrm{~mL})$ was added 3amino-3-(3-phenoxyphenyl)propanoic acid $(2077.46 \mathrm{mg}, 8.07 \mathrm{mmol}$ ) and sodium hydrogen carbonate (1356.55 mg, $16.15 \mathrm{mmol}$ ) in water $(6 \mathrm{~mL})$. The mixture was stirred at $90{ }^{\circ} \mathrm{C}$ for $12 \mathrm{~h}$. LCMS (595AB/1.5min): $\mathrm{RT}=0.903 \mathrm{~min},[\mathrm{M}+\mathrm{H}]^{+} 436.1$ showed $71 \%$ of $\mathrm{DP}$. The reaction mixture was adjusted to $\mathrm{pH}$ 4 with $2 \mathrm{HCl}$. The resulting solution was extracted with ether acetate $(100 \mathrm{~mL} \times 3)$ and the organic layers were combined to give 3-[4-(methylcarbamoyl)-2-nitro-anilino]-3-(3-phenoxyphenyl)propanoic acid $(3.6 \mathrm{~g}, 8.2677 \mathrm{mmol})$ as yellow solid.

\section{3-((2-amino-4-(methylcarbamoyl)phenyl)amino)-3-(3-phenoxyphenyl)propanoic acid (4)}




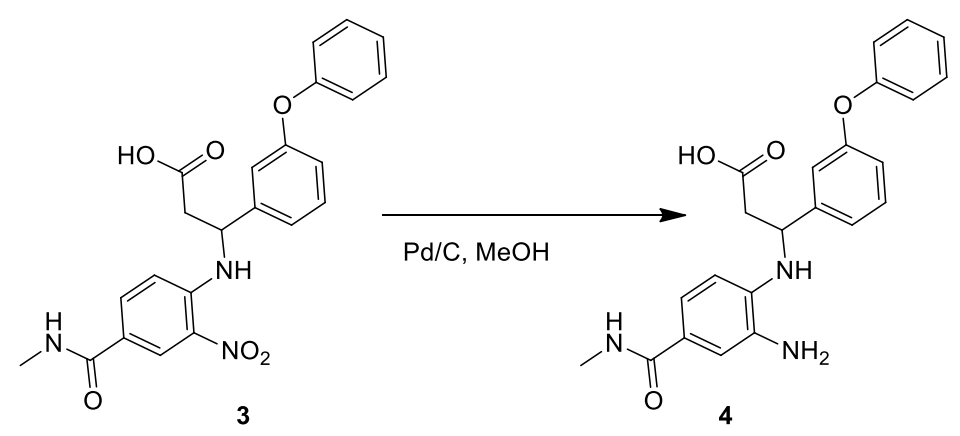

To a solution of 3-[4-(methylcarbamoyl)-2-nitro-anilino]-3-(3-phenoxyphenyl)propanoic acid (3.6 g, 8.27 $\mathrm{mmol})$ in methyl alcohol $(48 \mathrm{~mL})$ was added $\mathrm{Pd} / \mathrm{C}(1.2 \mathrm{~g}, 8.27 \mathrm{mmol})$. The mixture was stirred at $20^{\circ} \mathrm{C}$ for $3 \mathrm{~h}$ under $\mathrm{H}_{2}$ (50 psi). LCMS (5-95AB/1.5min): $\mathrm{RT}=0.632 \mathrm{~min},[\mathrm{M}+\mathrm{H}]^{+} 406.0$ showed $68 \%$ of DP. The mixture was filtered and concentrated to give 3-[2-amino-4-(methylcarbamoyl)anilino]-3-(3phenoxyphenyl)propanoic acid (3.2g, $5.3669 \mathrm{mmol}, 64.9 \%$ yield) as light yellow solid.

\section{3-[2-(5-ethoxy-3-formyl-2-hydroxy-phenyl)-5-(methylcarbamoyl)benzimidazol-1-yl]-3-(3-} phenoxyphenyl)propanoic acid (Compound 1)
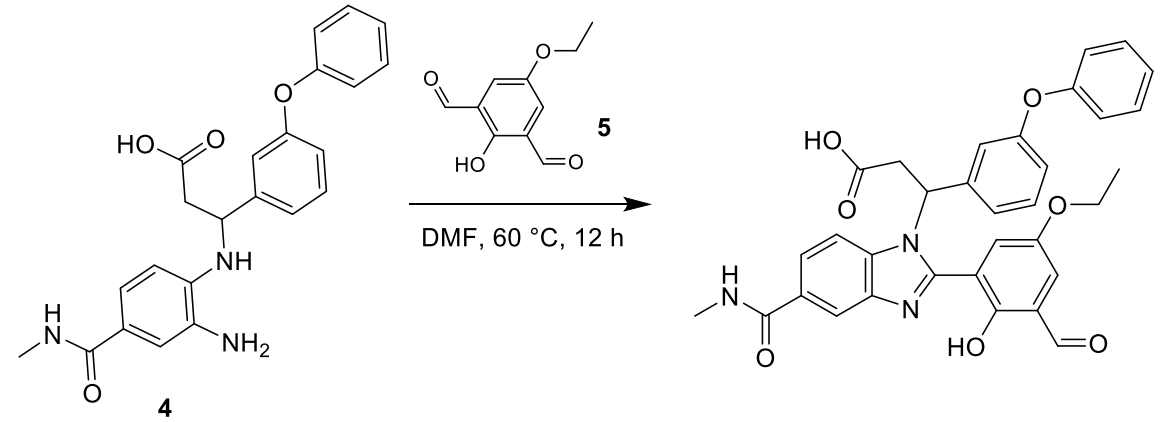

To a solution of 3-[2-amino-4-(methylcarbamoyl)anilino]-3-(3-phenoxyphenyl)propanoic acid (150.0 mg, $0.2600 \mathrm{mmol}$ ) in $\mathrm{N}, \mathrm{N}$-Dimethylformamide $(2 \mathrm{~mL})$ was added 5-ethoxy-2-hydroxy-benzene-1,3dicarbaldehyde $(80.46 \mathrm{mg}, 0.4100 \mathrm{mmol})$. The mixture was stirred at $60{ }^{\circ} \mathrm{C}$ for $12 \mathrm{~h}$. LCMS (595AB/1.5min): $\mathrm{RT}=0.912 \mathrm{~min},[\mathrm{M}+\mathrm{H}]^{+} 580.4$ showed $22 \%$ of DP. The crude product was purified by PrepHPLC with the following conditions: Column, [Luna C18 $150 \times 25,5 \mu \mathrm{m}$ ]; mobile phase: [ACN] and [water] (conditions: water(0.075\%TFA)-ACN); Detector: UV $254 \mathrm{~nm}$. RT: [time]. 3-[2-(5-ethoxy-3-formyl-2hydroxy-phenyl)-5-(methylcarba moyl)benzimidazol-1-yl]-3-(3-phenoxyphenyl)propanoic acid (72.8mg,0.1218mmol, $47 \%$ yield) was given as off-white solid, confirmed by LCMS (5-95AB/1.5min): RT = $0.725 \mathrm{~min},[\mathrm{M}+\mathrm{H}]^{+} 580.2$ showed $97 \%$ of DP. 
1H NMR (400 MHz, DMSO-d6) $\delta=10.18(\mathrm{~s}, 1 \mathrm{H}), 8.47(\mathrm{br} \mathrm{d}, \mathrm{J}=4.5 \mathrm{~Hz}, 1 \mathrm{H}), 8.21(\mathrm{~d}, \mathrm{~J}=1.2 \mathrm{~Hz}, 1 \mathrm{H}), 7.71$ $(\mathrm{dd}, \mathrm{J}=1.5,8.6 \mathrm{~Hz}, 1 \mathrm{H}), 7.54(\mathrm{~d}, \mathrm{~J}=3.2 \mathrm{~Hz}, 1 \mathrm{H}), 7.45-7.39(\mathrm{~m}, 2 \mathrm{H}), 7.38$ - $7.30(\mathrm{~m}, 3 \mathrm{H}), 7.14-7.09(\mathrm{~m}, 1 \mathrm{H})$, $7.05(\mathrm{~d}, \mathrm{~J}=7.8 \mathrm{~Hz}, 1 \mathrm{H}), 6.93-6.86(\mathrm{~m}, 4 \mathrm{H}), 5.87(\mathrm{t}, \mathrm{J}=7.3 \mathrm{~Hz}, 1 \mathrm{H}), 4.07(\mathrm{q}, \mathrm{J}=7.1 \mathrm{~Hz}, 2 \mathrm{H}), 3.74(\mathrm{dd}, \mathrm{J}=8.4$, $16.8 \mathrm{~Hz}, 1 \mathrm{H}), 3.34(\mathrm{dd}, \mathrm{J}=6.3,16.7 \mathrm{~Hz}, 1 \mathrm{H}), 2.82(\mathrm{~d}, \mathrm{~J}=4.4 \mathrm{~Hz}, 3 \mathrm{H}), 1.35(\mathrm{t}, \mathrm{J}=7.0 \mathrm{~Hz}, 3 \mathrm{H})$

\section{Compound 1 LCMS}

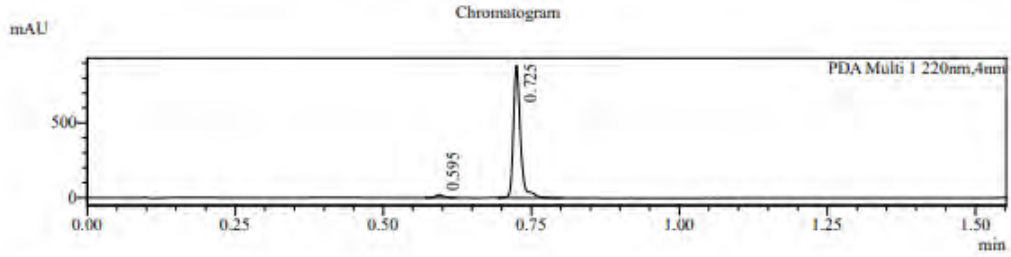

mAL

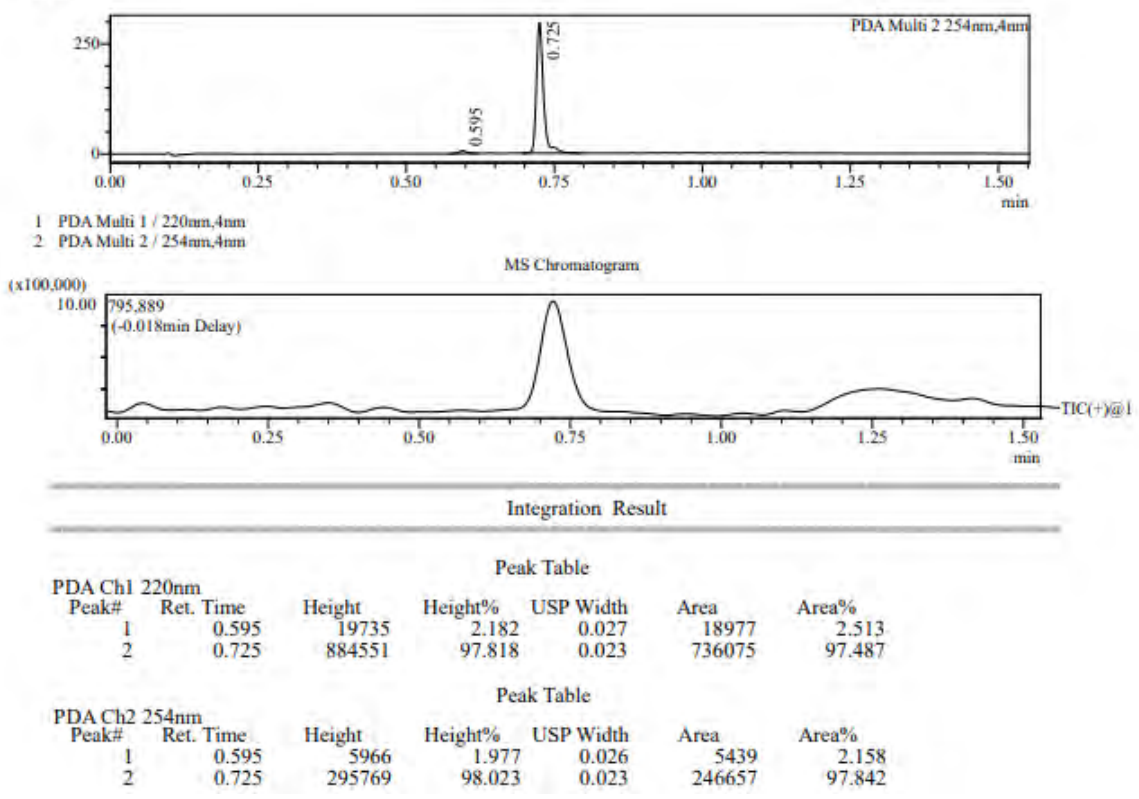



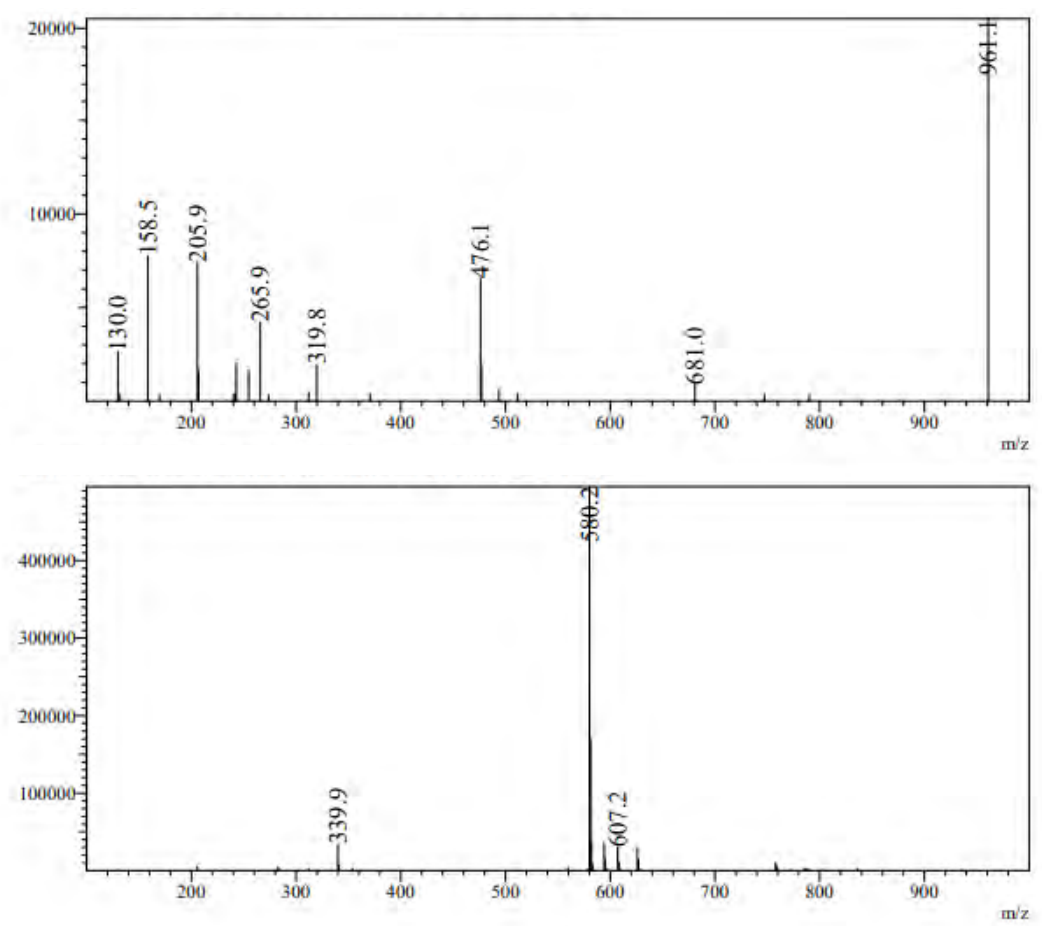

\section{Compound 1 1H NMR}

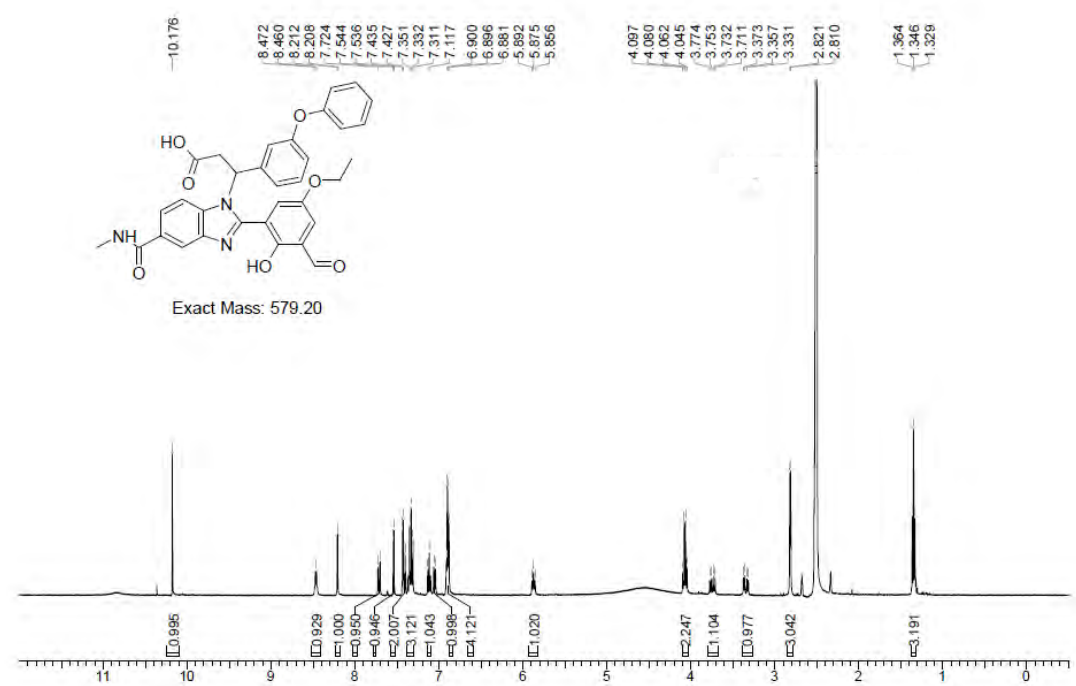

\section{Compound 2 and 3:}

4-hydroxy-5-methoxy-benzene-1, 3-dicarbaldehyde (3) 


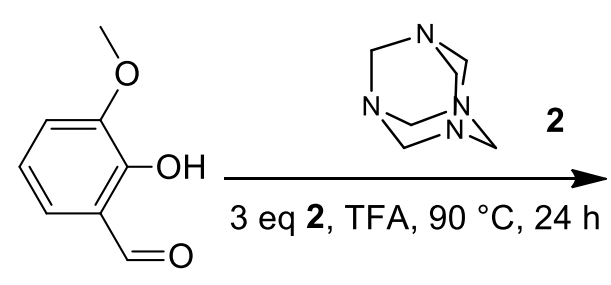

1

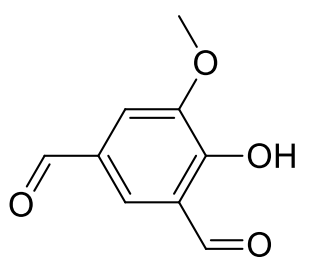

3

To a solution of hexamethylenetetramine $(6.91 \mathrm{~g}, 49.29 \mathrm{mmol})$ 3-hydroxy-2-methoxy-benzaldehyde (5.0 $\mathrm{g}, 32.86 \mathrm{mmol})$ in 2,2,2-trifluoroacetic acid $(50 \mathrm{~mL})$. The mixture was stirred at $90{ }^{\circ} \mathrm{C}$ for $12 \mathrm{~h}$ under $\mathrm{N}_{2}$. LCMS (5-95AB/1.5min): RT $=0.645 \mathrm{~min},[\mathrm{M}+\mathrm{H}]+181.0$ showed $20 \%$ of DP. The reaction mixture was diluted with $\mathrm{H}_{2} \mathrm{O}(250 \mathrm{~mL})$ extracted with EtOAc $(100 \mathrm{~mL} \times 2)$. The combined organic layer was dried over anhydrous $\mathrm{Na}_{2} \mathrm{SO}_{4}$ and concentrated in vacuum. Then purified by column chromatography $\left(\mathrm{SiO}_{2}, 15 \%\right.$ ethyl acetate in petroleum ether), TLC ( $25 \%$ ethyl acetate in petroleum ether, $R f=0.45$ ). Compound 4hydroxy-5-methoxy-benzene-1, 3-dicarbaldehyde (1.02 g, $5.6616 \mathrm{mmol}, 17.2 \%$ yield) was obtained as a colorless oil.

LCMS: GNEWX-1115-243-P1B, [M+H] ${ }^{+} 181.0$

\section{Synthesis of 4,5-dihydroxybenzene-1,3-dicarbaldehyde (4)}

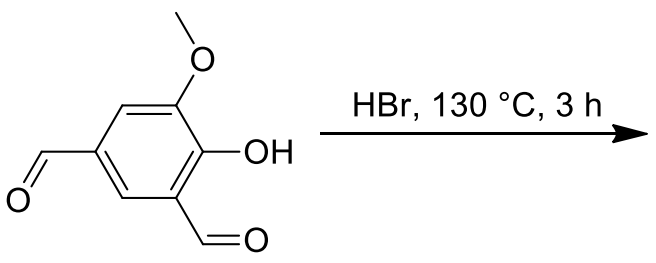

3

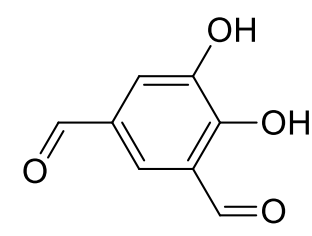

4

To a solution of 4-hydroxy-5-methoxy-benzene-1,3-dicarbaldehyde (900.0 mg, $5 \mathrm{mmol}$ ) in hydrobromic acid $(9 \mathrm{~mL})$ was added. The mixture was stirred at $130^{\circ} \mathrm{C}$ for $12 \mathrm{~h}$. LCMS $(5-95 \mathrm{AB} / 1.5 \mathrm{~min}): \mathrm{RT}=0.261 \mathrm{~min}$, $[\mathrm{M}+\mathrm{H}]+167.6$ showed $72 \%$ of DP. The reaction mixture was added ice water $(10 \mathrm{~mL})$ and diluted with $\mathrm{H}_{2} \mathrm{O}$ $(30 \mathrm{~mL})$ extracted with EtOAc $(30 \mathrm{~mL} \times 2)$. The combined organic layer was dried over anhydrous $\mathrm{Na}_{2} \mathrm{SO}_{4}$ and concentrated in vacuum to give the compound 4, 5-dihydroxybenzene-1,3-dicarbaldehyde (520 mg, $3.1301 \mathrm{mmol}, 62.7 \%$ yield) as a brown solid.

\section{3-[2-(5-formyl-2,3-dihydroxy-phenyl)-5-(methylcarbamoyl)benzimidazol-1-yl]-3-(3-}

phenoxyphenyl)propanoic acid (Compound 2) and 3-[2-(3-formyl-4,5-dihydroxy-phenyl)-5(methylcarbamoyl)benzimidazol-1-yl]-3-(3-phenoxyphenyl)propanoic acid (Compound 3) 


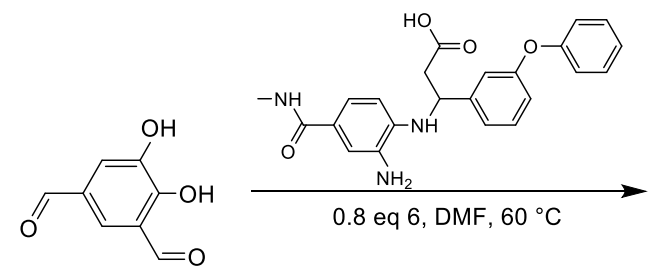

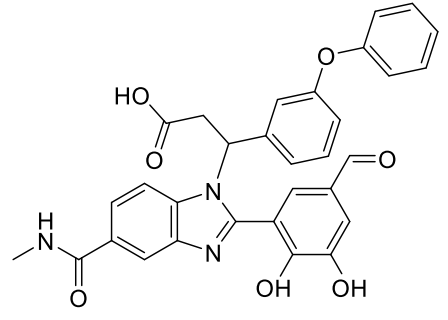

Compound 2

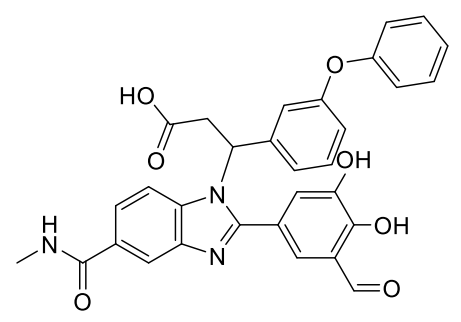

Compound 3

To a solution of 4, 5-dihydroxybenzene-1, 3-dicarbaldehyde $(50.0 \mathrm{mg}, 0.3000 \mathrm{mmol})$ and 3-[2-amino-4(methylcarbamoyl) anilino]-3-(3-phenoxyphenyl)propanoic acid (Compound 1 intermediate 4) (97.62 mg, $0.2400 \mathrm{mmol}$ ) in 1-methyl-2-pyrrolidinone $(1 \mathrm{~mL})$. The reaction mixture was stirred at $60{ }^{\circ} \mathrm{C}$ for $1 \mathrm{~h}$. LCMS (5-95AB/1.5min): RT =0.548 min, $[M+H]+552.3$ showed 40\% of DP1. LCMS (5-95AB/1.5min): RT =0.892 min, $[\mathrm{M}+\mathrm{H}]+552.3$ showed $13 \%$ of DP2. The reaction mixture was filtered and the filtrate was purified by prep-HPLC (Column Waters Xbridge $150 \times 25 \mathrm{~mm}, 5 \mu \mathrm{m}$; mobile phase: [water (0.025\%FA)-ACN]; B\%: 12\%42\%, $10 \mathrm{~min}$ ). The solution was lyophilized to give the compound 13-[2-(5-formyl-2,3-dihydroxy-phenyl)5-(methylcarbamoyl)benzimidazol-1-yl]-3-(3-phenoxyphenyl)propanoic acid (40 mg,0.0653 mmol, 21.7\% yield) as a yellow solid and compound 2 3-[2-(3-formyl-4,5-dihydroxy-phenyl)-5(methylcarbamoyl)benzimidazol-1-yl]-3-(3-phenoxyphenyl)propanoic acid $(5.3 \mathrm{mg}, 0.0090 \mathrm{mmol}, 3 \%$ yield) as a yellow solid.

LCMS: Compound 2 and $3,[\mathrm{M}+\mathrm{H}]^{+} 552.3$

DP 1LCMS (5-95AB/1.5min): RT =0.747 $\mathrm{min},[\mathrm{M}+\mathrm{H}]+552.3$ showed $94 \%$ of DP.

1H NMR (400 MHz, DMSO-d6) $\delta=10.30-10.27(\mathrm{~m}, 1 \mathrm{H}), 8.50-8.34(\mathrm{~m}, 1 \mathrm{H}), 8.21-8.09(\mathrm{~m}, 1 \mathrm{H}), 7.71-$ $7.65(m, 1 H), 7.48-7.29(m, 6 H), 7.14-7.08(m, 1 H), 7.02-6.91(m, 4 H), 6.86-6.82(m, 1 H), 6.24-6.09$ $(\mathrm{m}, 1 \mathrm{H}), 3.69-3.44(\mathrm{~m}, 2 \mathrm{H}), 2.84-2.80(\mathrm{~m}, 3 \mathrm{H})$

DP 2LCMS (5-95AB/1.5min): RT =0.732 $\mathrm{min},[\mathrm{M}+\mathrm{H}]+552.3$ showed $90 \%$ of DP.

1H NMR (400 MHz, DMSO-d6) $\delta=9.81-9.65(\mathrm{~m}, 1 \mathrm{H}), 8.48-8.36(\mathrm{~m}, 1 \mathrm{H}), 8.21-8.13(\mathrm{~m}, 1 \mathrm{H}), 7.69-7.60$ $(m, 1 H), 7.52$ - $7.44(m, 1 H), 7.42$ - $7.27(m, 5 H), 7.15$ - $7.08(m, 1 H), 7.05$ - $7.00(m, 1 H), 6.93$ - $6.79(m$, $4 H), 5.91-5.76(m, 1 H), 3.92-3.44(m, 2 H), 2.84-2.79(m, 3 H)$.

\section{Compound 2 LCMS}




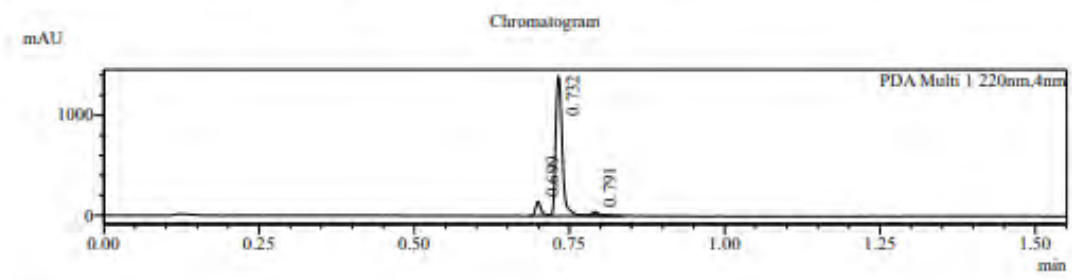

mNu

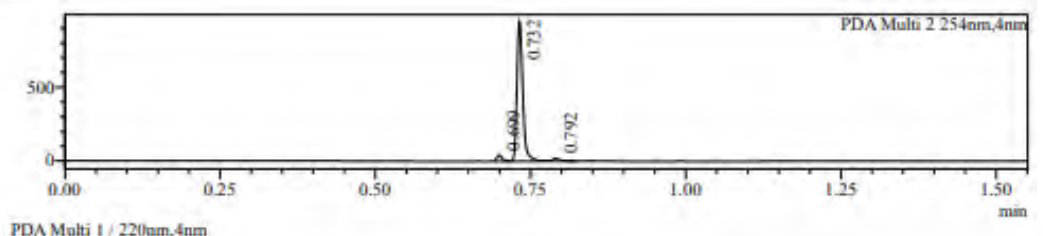

PDAMali $21 / 254$ an, 4 m
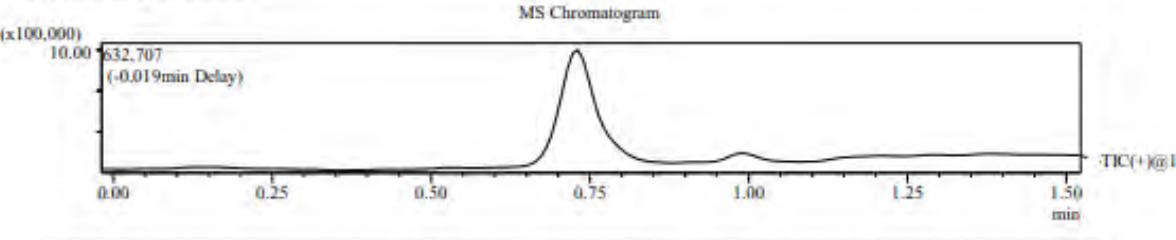

Integration Result

Peak Table

\begin{tabular}{|c|c|c|c|c|c|c|}
\hline $\mathrm{ACh}$ & 270 & & & & & \\
\hline & Time & Height & Height $\%$ & USP Width & Area & Area\% \\
\hline 1 & 0.699 & 143900 & 9.247 & 0.018 & 92216 & 7.932 \\
\hline 2 & 0.732 & 1379103 & 88.622 & 0.021 & 1046265 & 89.993 \\
\hline 3 & 0.791 & 33163 & 2.131 & 0.022 & 24123 & 2.075 \\
\hline
\end{tabular}

PDA Ch2 254nm Pcak Table

\begin{tabular}{rrrrrrr} 
PDACh2 & \multicolumn{1}{c}{ Height } & \multicolumn{1}{c}{ Height\% } & USP Width & \multicolumn{1}{c}{ Area } & \multicolumn{1}{c}{ Area\% } \\
Peak\# & Ret. Time & \multicolumn{1}{c}{ Height } \\
1 & 0.699 & 41780 & 4.173 & 0.018 & 26117 & 3.889 \\
2 & 0.732 & 941497 & 94.037 & 0.020 & 629639 & 93.748 \\
3 & 0.792 & 17923 & 1.790 & 0.024 & 15875 & 2.364
\end{tabular} 

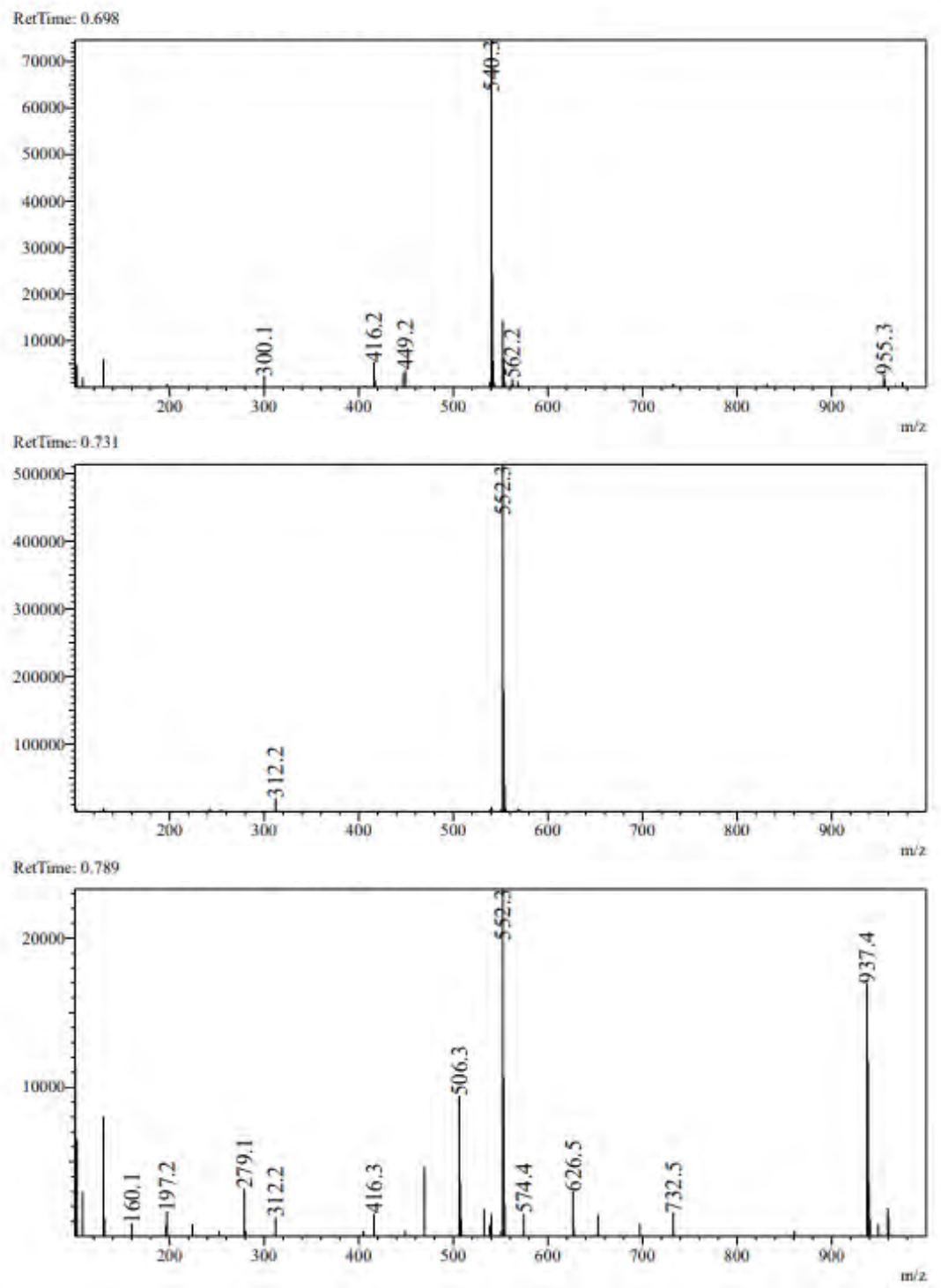

Compound 2 1H NMR 


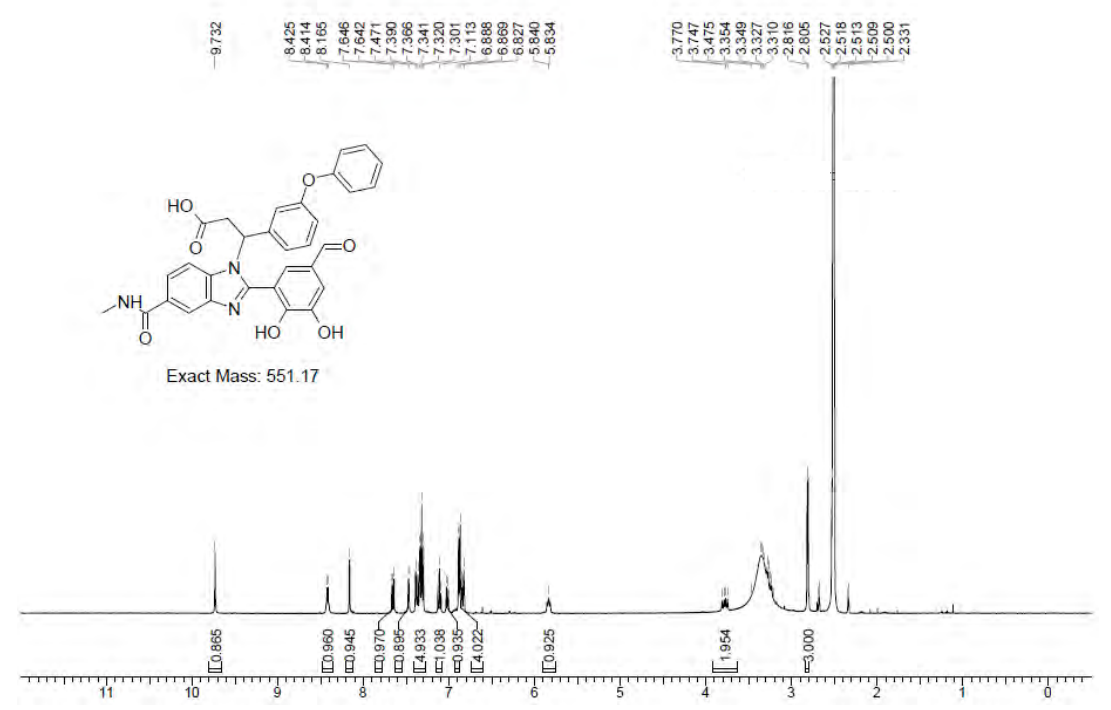

\section{Compound 3 LCMS}

$\mathrm{m} / \mathrm{L}$

Chrumatogran

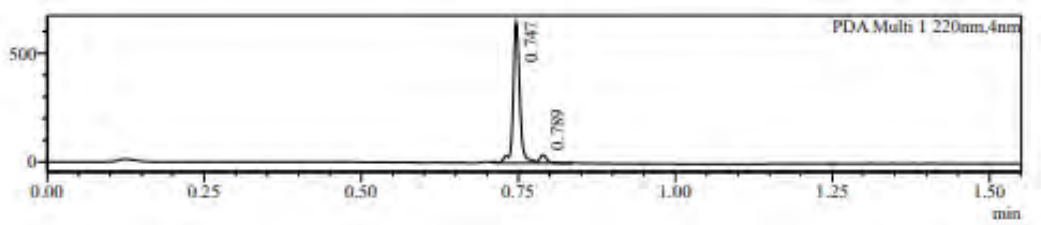

$\mathrm{mal}$

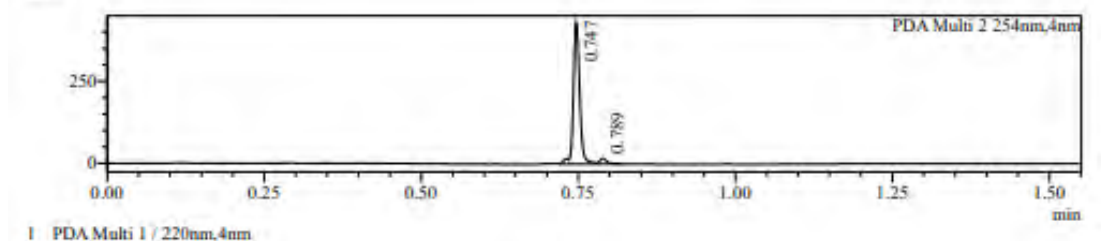

2 PDAMulti 2 $254 \mathrm{~mm} 4 \mathrm{~mm}$

c100,000) MS Chromatiogram

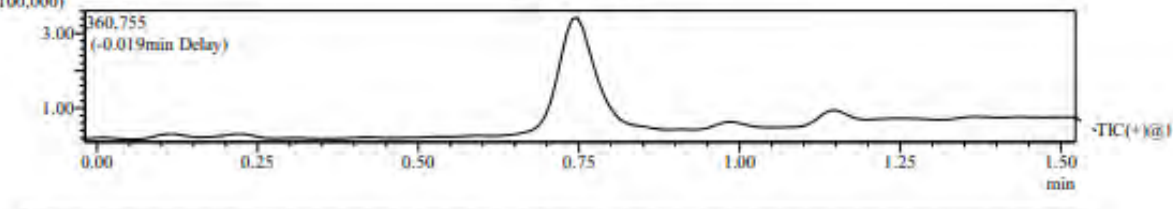

Integration Result

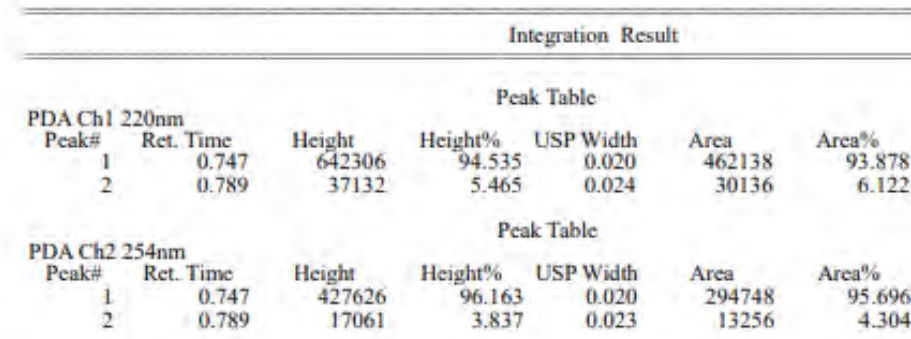



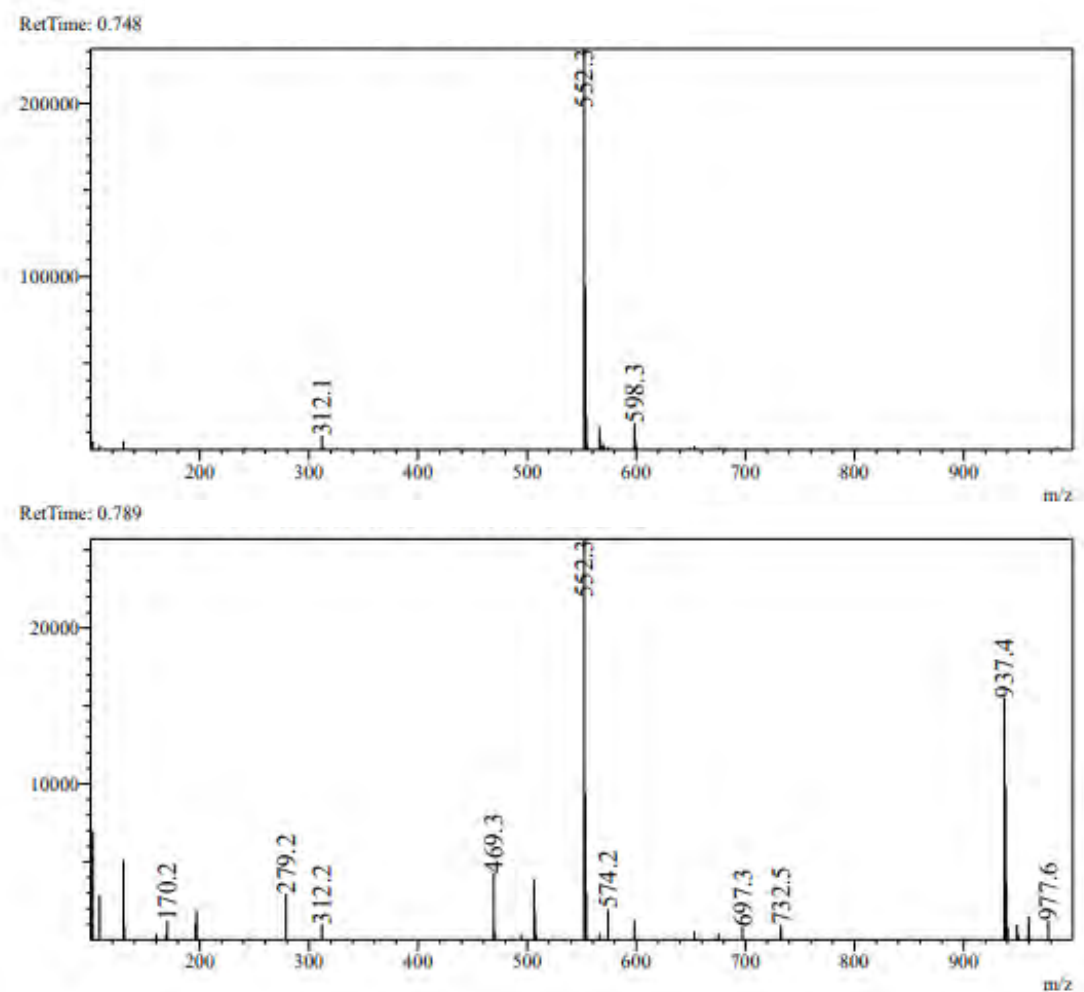

Compound 3 1H NMR

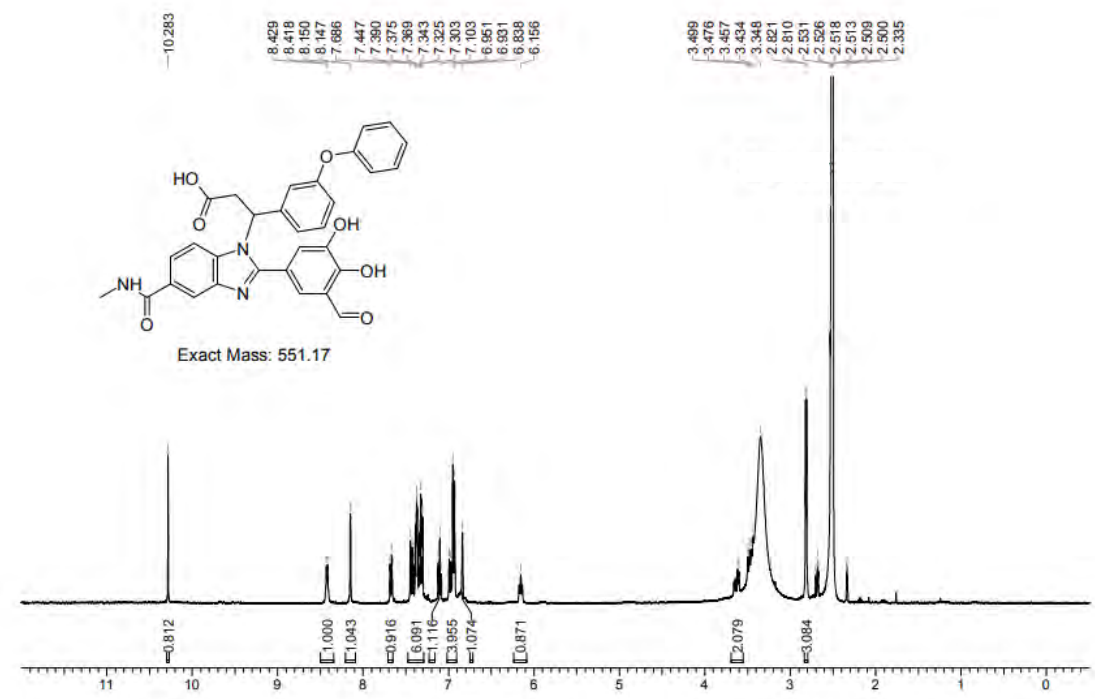

Compound 4:

3-(4-methoxyphenyl)-3-[4-(methylcarbamoyl)-2-nitro-anilino]propanoic acid (3) 
<smiles>CNC(=O)c1ccc(F)c([N+](=O)[O-])c1</smiles>

1

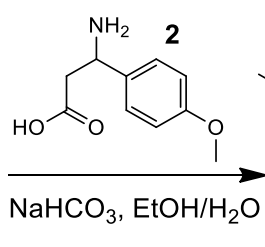

3

To a solution of 4-fluoro- $\mathrm{N}$-methyl-3-nitro-benzamide $(400.0 \mathrm{mg}, 2.02 \mathrm{mmol})$ in ethanol $(8 \mathrm{~mL})$ was added 3-(4-methoxyphenyl)-beta-alanine (394.09 mg, $2.02 \mathrm{mmol})$ and sodium hydrogen carbonate (339.14 $\mathrm{mg}$, $4.04 \mathrm{mmol}$ ) in water $(3 \mathrm{~mL})$. The mixture was stirred at $90{ }^{\circ} \mathrm{C}$ for $5 \mathrm{~h}$. LCMS (5-95AB/1.5min): RT $=0.834$ min, $[\mathrm{M}+\mathrm{H}]^{+} 374.2$ showed $88 \%$ of DP. The reaction mixture was adjusted to $\mathrm{pH} 4$ with $1 \mathrm{M} \mathrm{HCl}$. The resulting solution was extracted with ethyl acetate $(100 \mathrm{~mL} \times 3)$ and the organic layers were combined and dried to give 3-(4-methoxyphenyl)-3-[4-(methylcarbamoyl)-2-nitro-anilino]propanoic acid (740 mg, $1.8829 \mathrm{mmol}, 93.3 \%$ yield) as yellow solid. LCMS (5-95AB/1.5min): RT $=0.674 \mathrm{~min},[\mathrm{M}+\mathrm{H}]^{+} 373.9$ showed $95 \%$ of DP.

\section{3-[2-amino-4-(methylcarbamoyl)anilino]-3-(4-methoxyphenyl)propanoic acid (4)}<smiles>CNC(=O)c1ccc(NC(CC(=O)O)c2ccc(OC)cc2)c([N+](=O)[O-])c1</smiles>

3

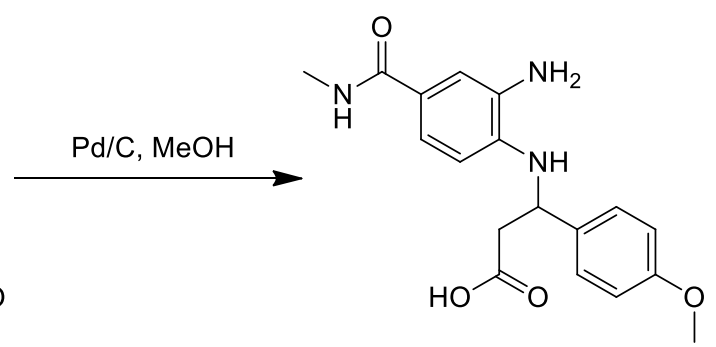

4

To a solution of 3-(4-methoxyphenyl)-3-[4-(methylcarbamoyl)-2-nitro-anilino]propanoic acid (700.0 mg, $1.78 \mathrm{mmol})$ in methyl alcohol $(15 \mathrm{~mL})$ was added $\mathrm{Pd} / \mathrm{C}(350.0 \mathrm{mg}, 1.78 \mathrm{mmol})$. The mixture was stirred at $20{ }^{\circ} \mathrm{C}$ for $6 \mathrm{~h}$ under $\mathrm{H}_{2}$ (15 psi). LCMS (5-95AB/1.5min): $\mathrm{RT}=0.524 \mathrm{~min},[\mathrm{M}+\mathrm{H}]^{+} 343.9$ showed $82 \%$ of DP. The mixture was filtered and concentrated to give 3-[2-amino-4-(methylcarbamoyl)anilino]-3-(4methoxyphenyl)propanoic acid (640 mg, $1.5283 \mathrm{mmol}, 85.8 \%$ yield) as purple solid.

3-[2-(5-ethoxy-3-formyl-2-hydroxy-phenyl)-5-(methylcarbamoyl)benzimidazol-1-yl]-3-(4methoxyphenyl)propanoic acid (Compound 4) 


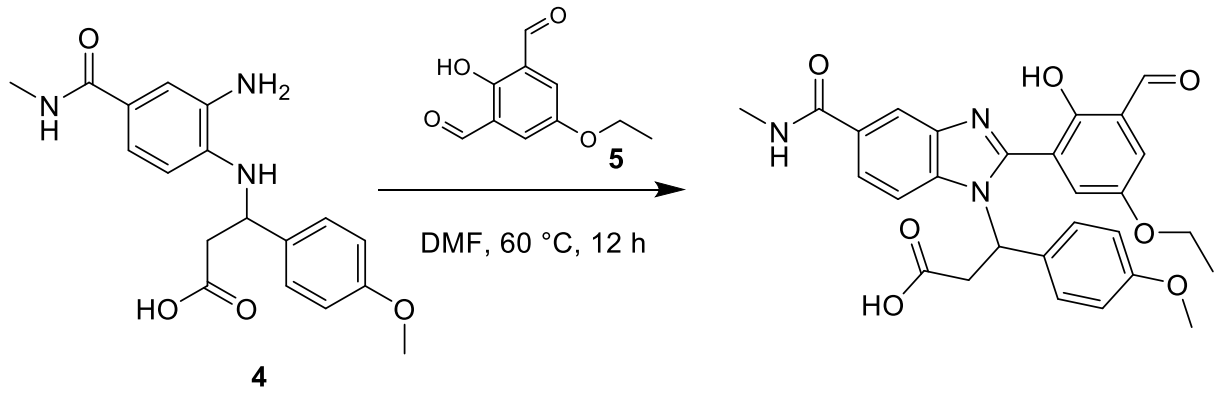

To a solution of 3-[2-amino-4-(methylcarbamoyl)anilino]-3-(4-methoxyphenyl)propanoic acid (80.0 mg, $0.1900 \mathrm{mmol}$ ) in $\mathrm{N}, \mathrm{N}$-Dimethylformamide $(1 \mathrm{~mL})$ was added 5-ethoxy-2-hydroxy-benzene-1,3dicarbaldehyde $(39.81 \mathrm{mg}, 0.2100 \mathrm{mmol})$. The mixture was stirred at $60{ }^{\circ} \mathrm{C}$ for $12 \mathrm{~h}$. LCMS (595AB/1.5min): $R T=0.811 \mathrm{~min},[\mathrm{M}+\mathrm{H}]^{+} 518.2$ showed $30 \%$ of DP. The crude product was purified by PrepHPLC with the following conditions: Column, [Phenomenex Synergi C18 $150 \times 25 \mathrm{~mm}, 10 \mu \mathrm{m}$ ]; mobile phase: [ACN] and [water] (conditions: water(0.1\%TFA)-ACN); Detector: UV 254 nm. RT: [10 min]. 3-[2-(5ethoxy-3-formyl-2-hydroxy-phenyl)-5-(methylcarbamoyl)benzimidazol-1-yl]-3-(4-

methoxyphenyl)propanoic acid (25.1 $\mathrm{mg}, 0.0485 \mathrm{mmol}$, 26\% yield) was given as yellow solid. LCMS (595AB/1.5min): $\mathrm{RT}=0.648 \mathrm{~min},[\mathrm{M}+\mathrm{H}]^{+} 518.1$ showed $100 \%$ of DP.

1H NMR (400 MHz, DMSO-d6) $\delta=10.20(\mathrm{~s}, 1 \mathrm{H}), 8.46-8.38(\mathrm{~m}, 1 \mathrm{H}), 8.19$ (d, J = 1.2 Hz, 1H), 7.67 (dd, J = 1.5, 8.6 Hz, 1H), $7.55(d, J=3.2 \mathrm{~Hz}, 1 \mathrm{H}), 7.50(\mathrm{~d}, \mathrm{~J}=3.2 \mathrm{~Hz}, 1 \mathrm{H}), 7.37(\mathrm{~d}, \mathrm{~J}=8.6 \mathrm{~Hz}, 1 \mathrm{H}), 7.19(\mathrm{~d}, \mathrm{~J}=8.7 \mathrm{~Hz}$, $2 \mathrm{H}), 6.88(\mathrm{~d}, \mathrm{~J}=8.9 \mathrm{~Hz}, 2 \mathrm{H}), 5.84(\mathrm{dd}, \mathrm{J}=6.2,8.6 \mathrm{~Hz}, 1 \mathrm{H}), 4.09(\mathrm{q}, \mathrm{J}=7.0 \mathrm{~Hz}, 2 \mathrm{H}), 3.71(\mathrm{~s}, 3 \mathrm{H}), 3.68(\mathrm{dd}, 1 \mathrm{H})$, $3.32(\mathrm{dd}, \mathrm{J}=6.0,16.6 \mathrm{~Hz}, 1 \mathrm{H}), 2.80(\mathrm{~d}, \mathrm{~J}=4.4 \mathrm{~Hz}, 3 \mathrm{H}), 1.35(\mathrm{t}, \mathrm{J}=6.9 \mathrm{~Hz}, 3 \mathrm{H})$

\section{Compound 4 LCMS}



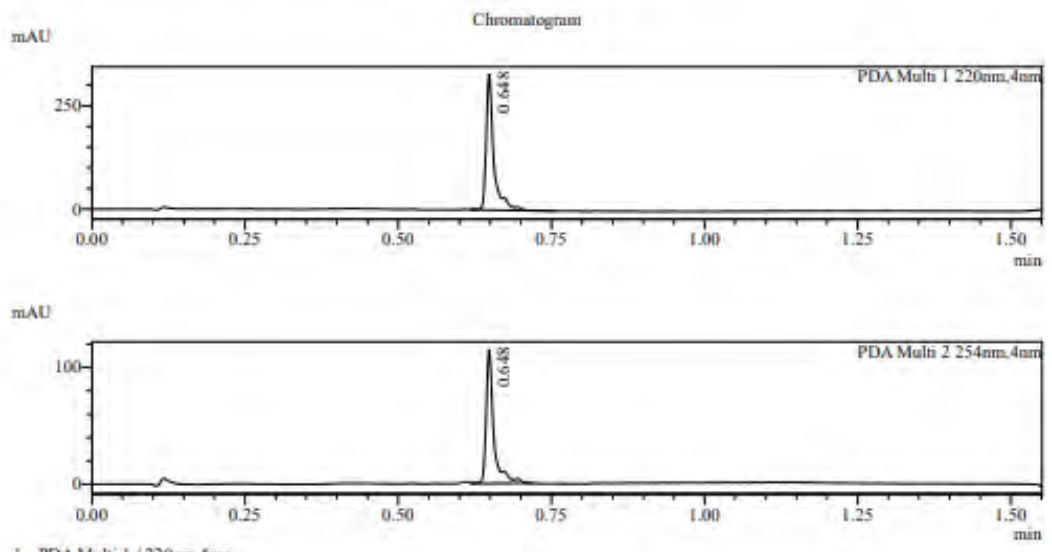

1 PDN Man i 220andan

(x100,000) MS Chromatogram

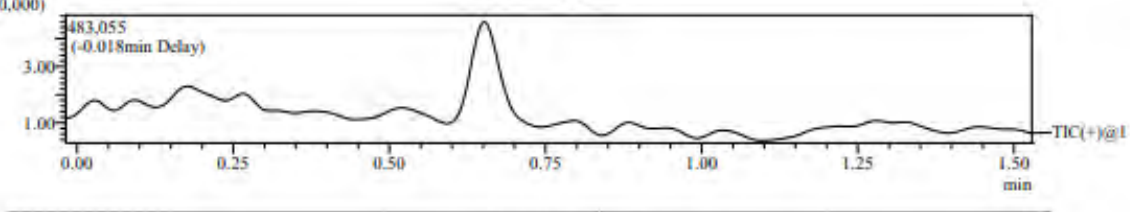

\begin{tabular}{l}
\hline \hline Integration Result \\
\hline \hline
\end{tabular}

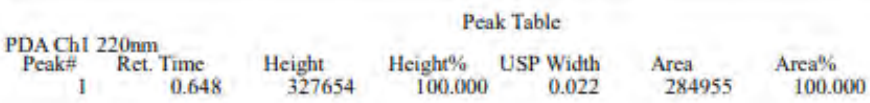
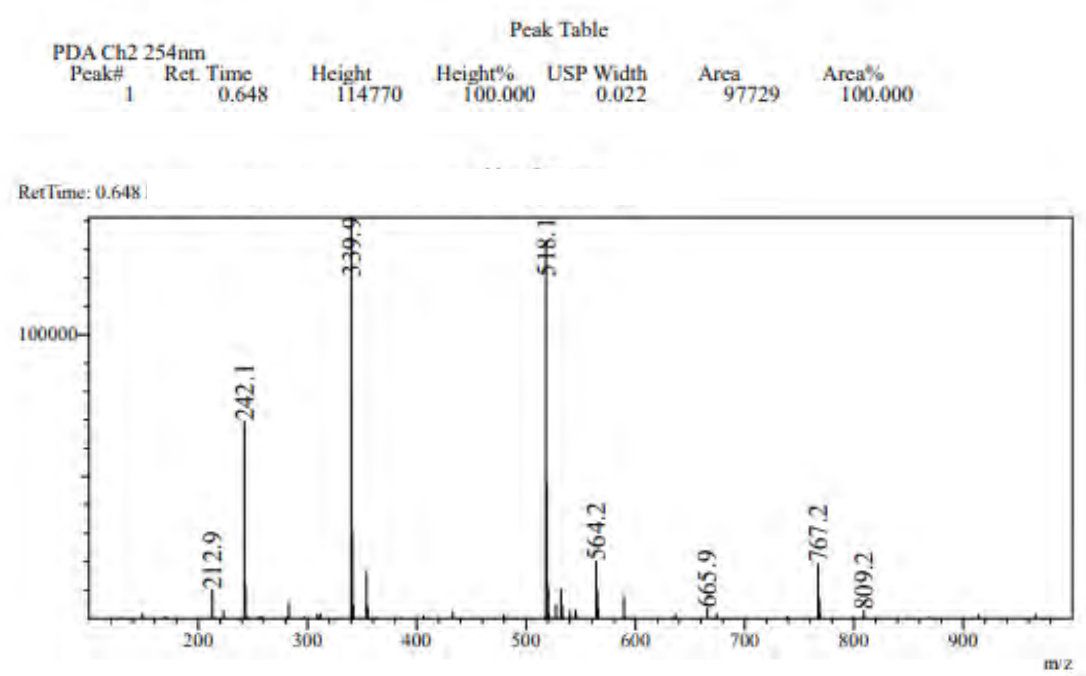

\section{Compound 4 1H NMR}




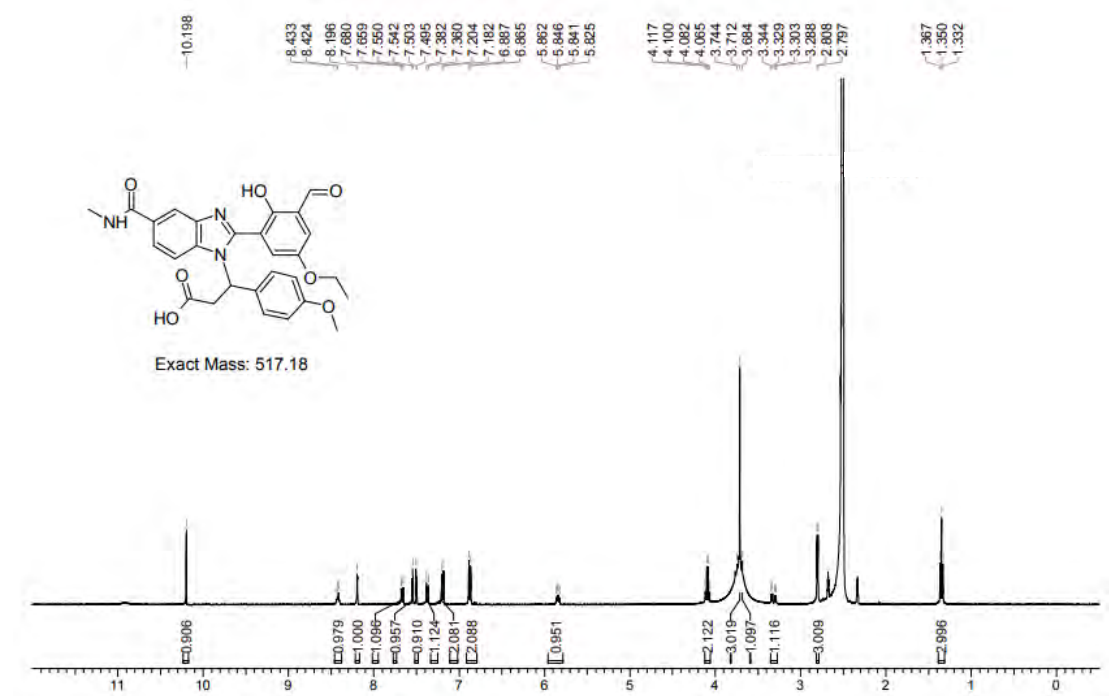

\section{Compound 5:}

3-(4-fluorophenyl)-3-[4-(methylcarbamoyl)-2-nitro-anilino]propanoic acid (4)<smiles>CNC(=O)c1ccc(F)c([N+](=O)[O-])c1</smiles>

To a solution of 4-fluoro- $\mathrm{N}$-methyl-3-nitro-benzamide $(400.0 \mathrm{mg}, 2.02 \mathrm{mmol})$ dissolved in ethanol (10 mL) and water (2 mL), 3-amino-3-(4-fluorophenyl)propanoic acid (443.74 mg, $2.42 \mathrm{mmol}$ ) and sodiumbicarbonate $(343.23 \mathrm{mg}, 4.04 \mathrm{mmol})$ were added. The mixture was stirred at $80^{\circ} \mathrm{C}$ for $12 \mathrm{~h}$. LCMS (5-95AB/1.5min): $\mathrm{RT}=0.845 \mathrm{~min},[\mathrm{M}+\mathrm{H}]^{+} 362.2$ showed $66 \%$ of DP. The reaction mixture was diluted with water $(50 \mathrm{~mL})$ and extracted with ethyl acetate $(30 \mathrm{~mL} \times 3)$. The combined organic layers were washed with brine $(30 \mathrm{~mL} \times 3)$, dried over $\mathrm{Na}_{2} \mathrm{SO}_{4}$, filtered and concentrated under reduced pressure to give a residue. 3-(4-fluorophenyl)-3-[4-(methylcarbamoyl)-2-nitro-anilino]propanoic acid (700 mg, 1.8986 mmol, $94.1 \%$ yield) was given as light yellow solid, which confirmed by LCMS (5-95AB/1.5min): RT = 0.835 $\min ,[\mathrm{M}+\mathrm{H}]^{+} 362.2$ showed $98 \%$ of DP.

\section{3-[2-amino-4-(methylcarbamoyl)anilino]-3-(4-fluorophenyl)propanoic acid (5)}




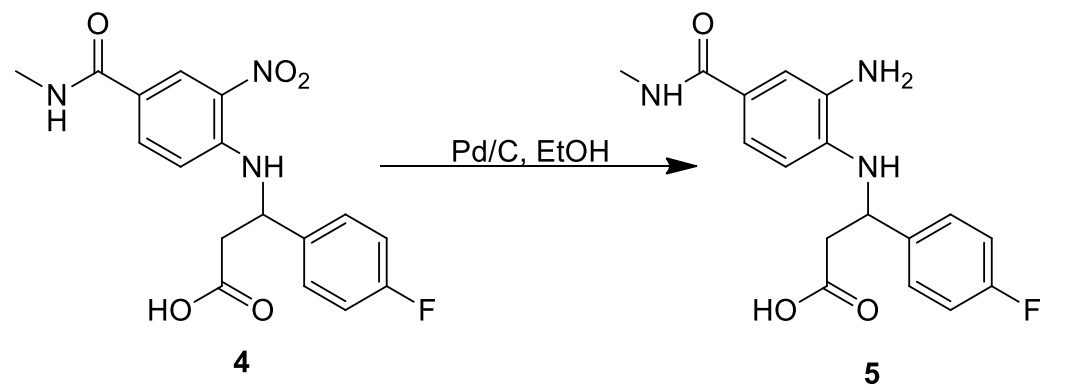

To a solution of 3-(4-fluorophenyl)-3-[4-(methylcarbamoyl)-2-nitro-anilino]propanoic acid (714.29 mg, $1.94 \mathrm{mmol})$ in methyl alcohol $(15.306 \mathrm{~mL}$ ) was added Pd/C (350 mg, $1.94 \mathrm{mmol})$. The mixture was stirred at $20^{\circ} \mathrm{C}$ for $5 \mathrm{~h}$ under $\mathrm{H}_{2}$ (15 psi). LCMS (5-95AB/1.5min): RT = $0.531 \mathrm{~min},[\mathrm{M}+\mathrm{H}]^{+} 331.9$ showed $94 \%$ of DP. The mixture was filtered and concentrated to give 3-[2-amino-4-(methylcarbamoyl)anilino]-3-(4fluorophenyl)propanoic acid (630 mg,1.7683 mmol, 91.3\% yield) as purple solid, confirmed by LCMS (595AB/1.5min): $\mathrm{RT}=0.528 \mathrm{~min},[\mathrm{M}+\mathrm{H}]^{+} 331.9$ showed $93 \%$ of $D P$.

\section{5-ethoxy-2-hydroxy-benzene-1,3-dicarbaldehyde (7)}

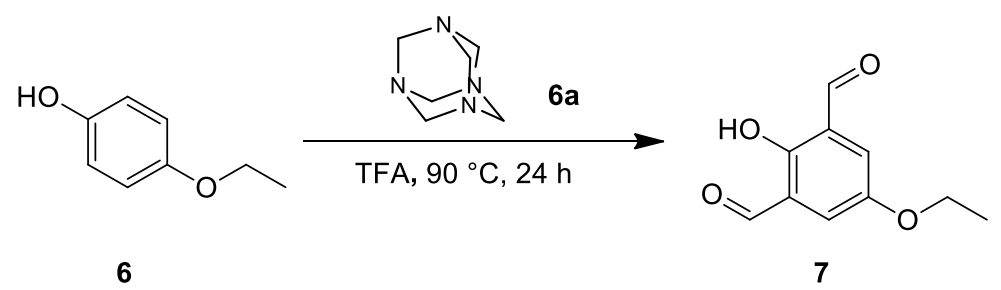

To a solution of 4-ethoxyphenol (10.0 g, $72.38 \mathrm{mmol})$ in 2,2,2-trifluoroacetic acid (100 mL) was added hexamethylenetetramine $(20.29 \mathrm{~g}, 144.76 \mathrm{mmol})$. The mixture was stirred at $90{ }^{\circ} \mathrm{C}$ for $48 \mathrm{~h}$ under $\mathrm{N}_{2}$. LCMS (5-95AB/1.5min): RT = 0.0.787 $\mathrm{min},[\mathrm{M}+\mathrm{H}]^{+} 195.1$ showed $45 \%$ of DP. The mixture was poured into $3 \mathrm{M} \mathrm{HCl}(60 \mathrm{~mL})$ and stirred for $10 \mathrm{~min}$, after which it was extracted with $\mathrm{DCM}(100 \mathrm{~mL} \times 3)$. The crude product was purified by silica gel column with petroleum ether to give 5-ethoxy-2-hydroxy-benzaldehyde (1.2 g, $7.2215 \mathrm{mmol}, 10 \%$ yield) as light yellow solid. 5-ethoxy-2-hydroxy-benzene-1,3-dicarbaldehyde (5.5 g, $28.324 \mathrm{mmol}$, 39.1\% yield) was given as light yellow solid, confirmed by HNMR: $1 \mathrm{H}$ NMR (400 MHz, CHLOROFORM-d) $\delta=11.05(\mathrm{~s}, 1 \mathrm{H}), 10.14(\mathrm{~s}, 2 \mathrm{H}), 7.43(\mathrm{~s}, 2 \mathrm{H}), 4.00(\mathrm{q}, \mathrm{J}=7.0 \mathrm{~Hz}, 2 \mathrm{H}), 1.36$ (t, J = 7.0 Hz, $3 \mathrm{H})$.

3-[2-(5-ethoxy-3-formyl-2-hydroxy-phenyl)-5-(methylcarbamoyl)benzimidazol-1-yl]-3-(4fluorophenyl)propanoic acid (Compound 5) 


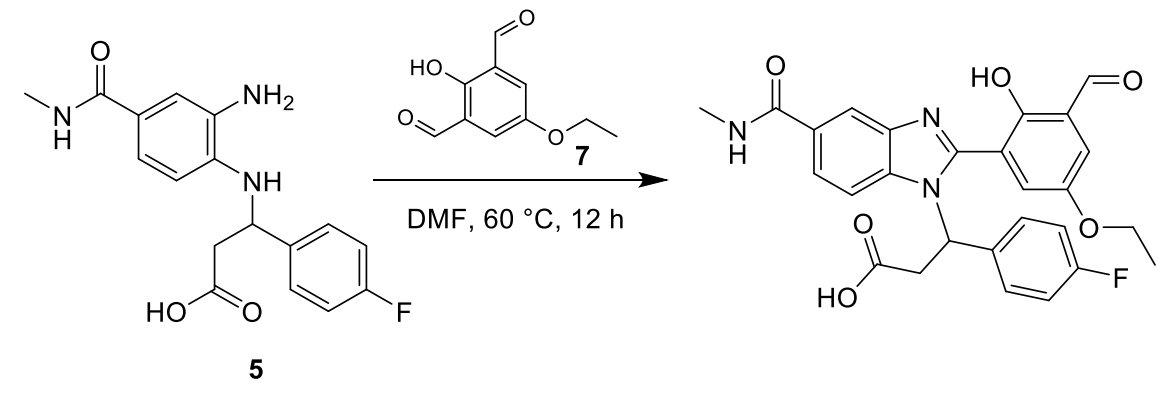

To a solution of 3-[2-amino-4-(methylcarbamoyl)anilino]-3-(4-fluorophenyl)propanoic acid (80.0 mg, $0.2300 \mathrm{mmol}$ ) in $\mathrm{N}, \mathrm{N}$-dimethylformamide $(1 \mathrm{~mL})$ was added 5-ethoxy-2-hydroxy-benzene-1,3dicarbaldehyde $(48.48 \mathrm{mg}, 0.2500 \mathrm{mmol})$. The mixture was stirred at $60{ }^{\circ} \mathrm{C}$ for $12 \mathrm{~h}$. LCMS (595AB/1.5min): $\mathrm{RT}=0.826 \mathrm{~min},[\mathrm{M}+\mathrm{H}]^{+} 506.1$ showed $59 \%$ of DP. The crude product was purified by PrepHPLC with the following conditions: Column, [Phenomenex Synergi C18 $150 \times 25 \mathrm{~mm}, 10 \mu \mathrm{m}$ ]; mobile phase: [ACN] and [water] (conditions: water(0.225\%FA)-ACN); Detector, UV $254 \mathrm{~nm} . \mathrm{RT}$ :[10 min]. 3-[2-(5ethoxy-3-formyl-2-hydroxy-phenyl)-5-(methylcarbamoyl)benzimidazol-1-yl]-3-(4-fluorophenyl)propanoic acid (19.3 mg, $0.0370 \mathrm{mmol}, 16.3 \%$ yield) was given as yellow solid, confirmed by LCMS (5-95AB/1.5min): $\mathrm{RT}=0.667 \mathrm{~min},[\mathrm{M}+\mathrm{H}]^{+} 506.1$ showed $97 \%$ of DP.

1H NMR (400 MHz, METHANOL-d4) $\delta=10.04(\mathrm{~s}, 1 \mathrm{H}), 8.21(\mathrm{~d}, \mathrm{~J}=1.1 \mathrm{~Hz}, 1 \mathrm{H}), 7.70$ (dd, J = 1.6, 8.7 Hz, 1H), $7.57(\mathrm{~d}, \mathrm{~J}=3.1 \mathrm{~Hz}, 1 \mathrm{H}), 7.49(\mathrm{~d}, \mathrm{~J}=3.2 \mathrm{~Hz}, 1 \mathrm{H}), 7.38-7.28(\mathrm{~m}, 3 \mathrm{H}), 7.08(\mathrm{t}, \mathrm{J}=8.7 \mathrm{~Hz}, 2 \mathrm{H}), 6.03(\mathrm{t}, \mathrm{J}=7.3$ $\mathrm{Hz}, 1 \mathrm{H}), 4.14(\mathrm{q}, \mathrm{J}=7.1 \mathrm{~Hz}, 2 \mathrm{H}), 3.68(\mathrm{dd}, \mathrm{J}=7.9,16.3 \mathrm{~Hz}, 1 \mathrm{H}), 3.40-3.35(\mathrm{~m}, 1 \mathrm{H}), 2.97-2.94(\mathrm{~s}, 3 \mathrm{H}), 1.44$ $(t, J=7.0 \mathrm{~Hz}, 3 \mathrm{H})$

\section{Compound 5 LCMS}


mAU

Chromatogram

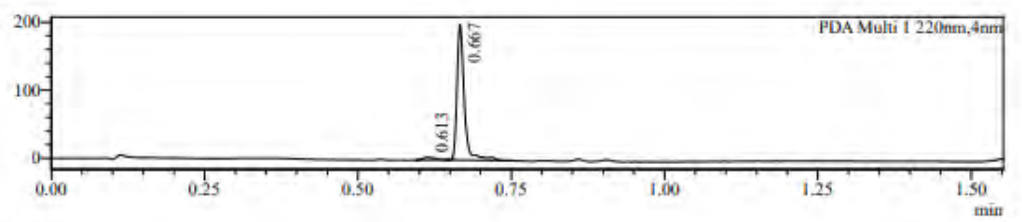

MAU

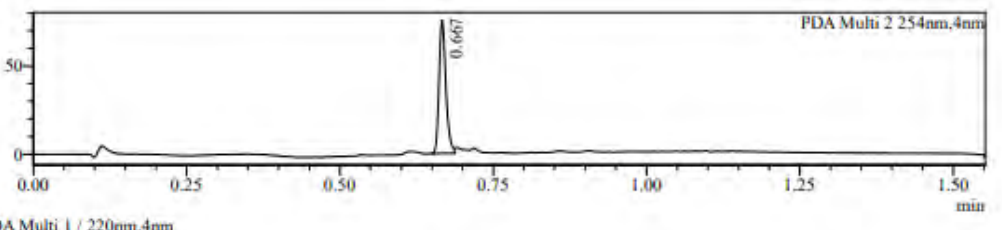

1 PDA Multi 1/ 220nm, $4 \mathrm{~nm}$

(x100,000) MS Chromatogran

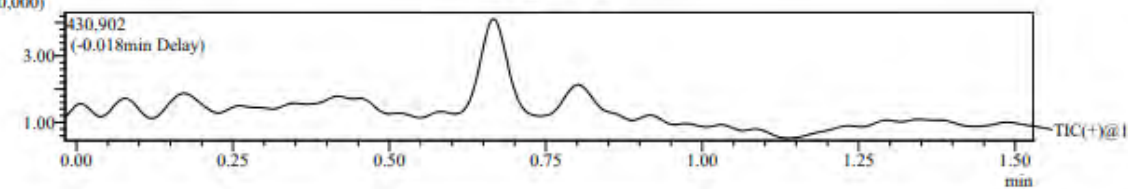

Integration Result

\begin{tabular}{|c|c|c|c|c|c|c|}
\hline & & \multicolumn{5}{|c|}{ Integration Result } \\
\hline \multicolumn{7}{|c|}{ PDAChl $220 \mathrm{~nm}$} \\
\hline Peak\# & Ret. Time & Height & Height $\%$ & USP Width & Area & Area $\%$ \\
\hline 1 & 0.613 & 3820 & 1.884 & 0.037 & 5075 & 2.970 \\
\hline 2 & 0.667 & 198944 & 98.116 & 0.023 & 165837 & 97.030 \\
\hline
\end{tabular}

Peak Table

$\underset{\text { Peak\# }}{\operatorname{PDACh} 254 \mathrm{~nm}} \underset{\text { Ret. Time }}{2}$ Height $\quad$ Height $\%$ USP Width Area Area\%

$\begin{array}{rrrrrrr}\text { Peak\# } & \text { Ret. Time } & \text { Height } & \text { Height } \% & \text { USP Width } & \text { Area } & \text { Area\% } \\ 1 & 0.667 & 75355 & 100.000 & 0.023 & 58642 & 100.000\end{array}$
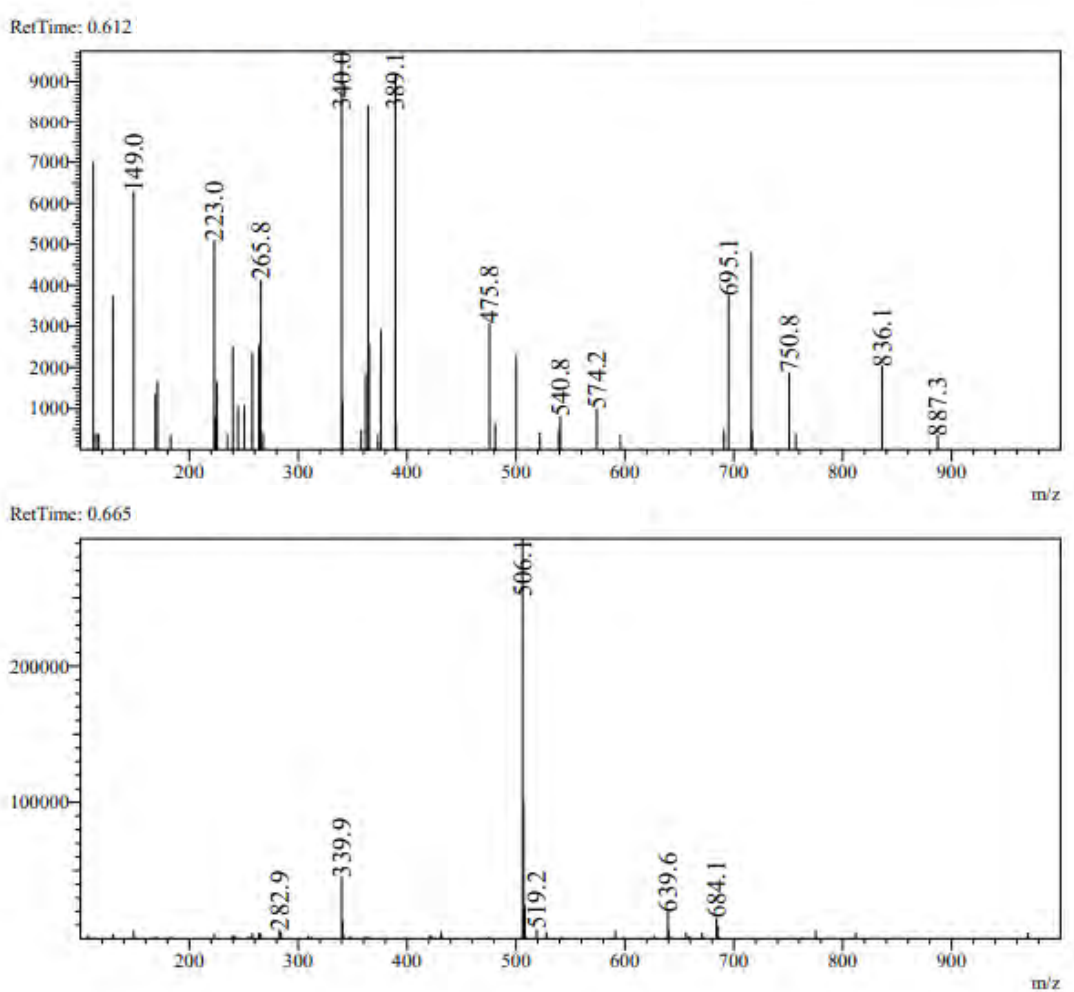


\section{Compound 5 1H NMR}

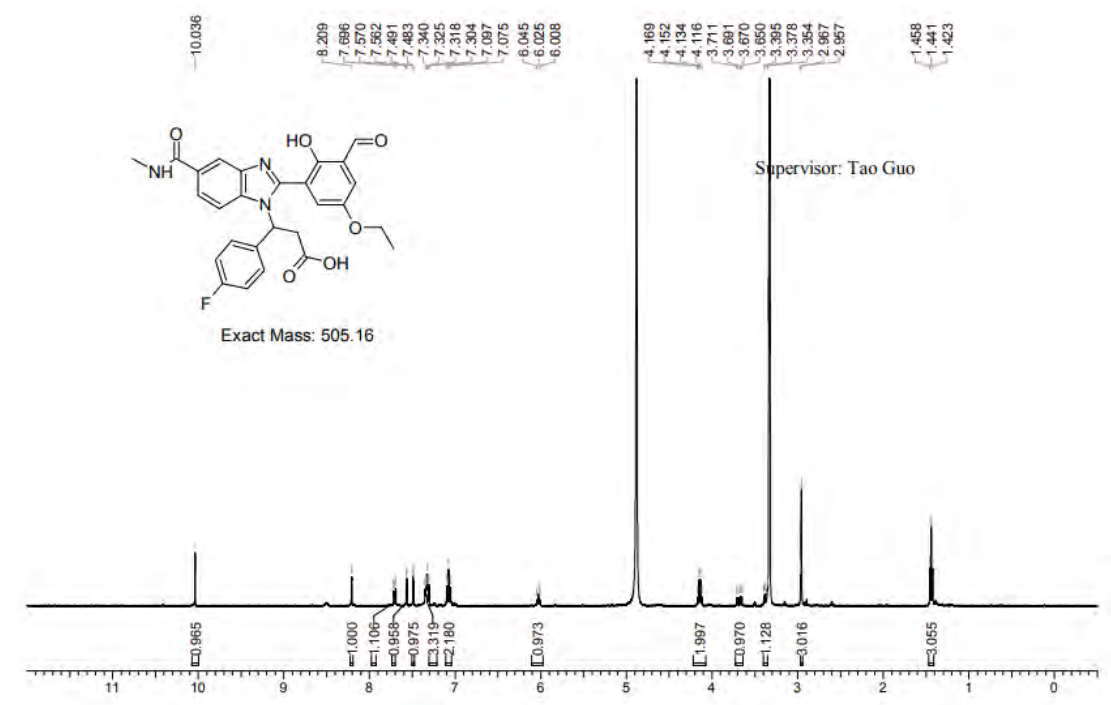

\section{Compound 6:}

3-[2-(2,4-dichloro-6-hydroxy-phenyl)-5-(methylcarbamoyl)benzimidazol-1-yl]-3-(3phenoxyphenyl)propanoic acid (Compound 6)
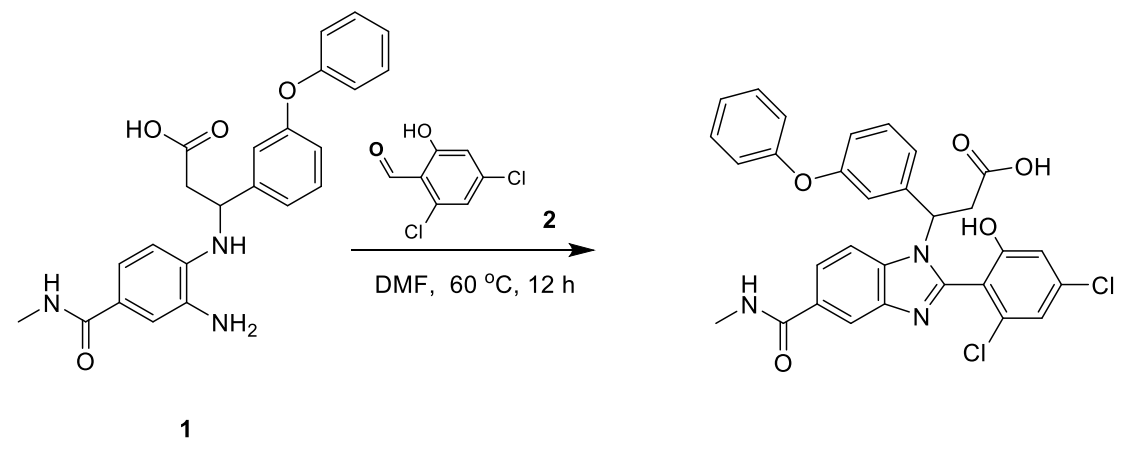

To a solution of 3-[2-amino-4-(methylcarbamoyl)anilino]-3-(3-phenoxyphenyl)propanoic acid (150.0 mg, $0.2600 \mathrm{mmol}$ ) in $\mathrm{N}, \mathrm{N}$-Dimethylformamide $(3 \mathrm{~mL}$ ) was added 2,4-dichloro-6-hydroxybenzaldehyde (64.31 $\mathrm{mg}, 0.3400 \mathrm{mmol})$. The mixture was stirred at $60^{\circ} \mathrm{C}$ for $12 \mathrm{~h}$. LCMS $(5-95 \mathrm{AB} / 1.5 \mathrm{~min}): \mathrm{RT}=0.910 \mathrm{~min}$, $[\mathrm{M}+\mathrm{H}]^{+} 576.2$ showed $31 \%$ of DP. The crude product was purified by Prep-HPLC with the following conditions: Column, [Phenomenex Synergi C18 $150 \times 25 \mathrm{~mm}, 10 \mu \mathrm{m}$ ]; mobile phase: [ACN] and [water] (conditions: water(0.225\%FA)-ACN); Detector, UV $254 \mathrm{~nm}$. RT: [10 min]. 3-[2-(2,4-dichloro-6-hydroxyphenyl)-5-(methylcarbamoyl)benzimidazol-1-yl]-3-(3-phenoxyphenyl)propanoic acid (18.1 mg, 0.0314 mmol, $12.1 \%$ yield) was given as off-white solid. LCMS (5-95AB/1.5min): RT $=0.741 \mathrm{~min},[\mathrm{M}+\mathrm{H}]^{+} 576.0$ showed $100 \%$ of DP. 
1H NMR (400 MHz, methanol-d4) $\delta=8.20(\mathrm{~s}, 1 \mathrm{H}), 7.74-7.67(\mathrm{~m}, 1 \mathrm{H}), 7.35(\mathrm{td}, \mathrm{J}=7.8,15.2 \mathrm{~Hz}, 1 \mathrm{H}), 7.28$

- $7.20(\mathrm{~m}, 3 \mathrm{H}), 7.20$ - $7.12(\mathrm{~m}, 2 \mathrm{H}), 7.10$ - $7.03(\mathrm{~m}, 1 \mathrm{H}), 6.99(\mathrm{br} \mathrm{dd}, \mathrm{J}=2.0,3.8 \mathrm{~Hz}, 2 \mathrm{H}), 6.92$ - $6.75(\mathrm{~m}, 3 \mathrm{H})$, $5.94-5.74(m, 1 H), 3.81-3.65(m, 1 H), 3.26-3.10(m, 1 H), 2.97(s, 3 H)$.

\section{Compound 6 LCMS}
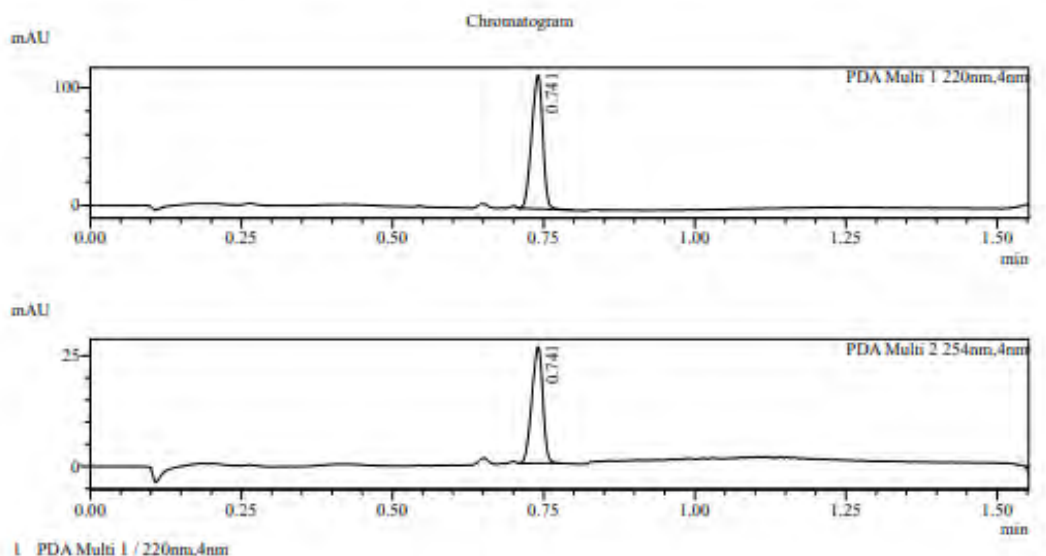

2 PDA Multi 2/254nm,4nm

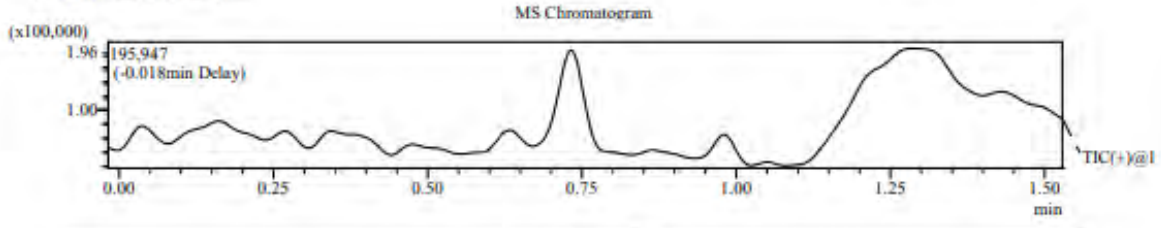

Integration Result

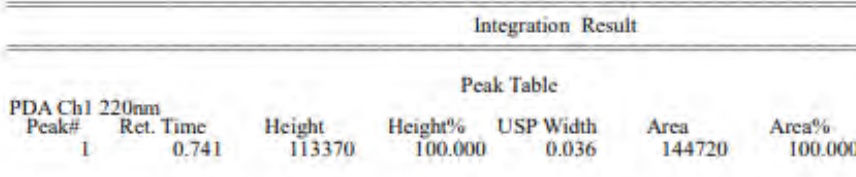

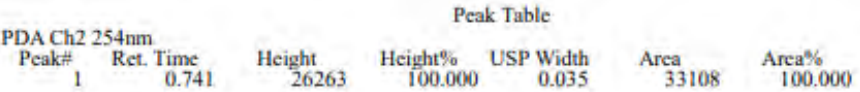

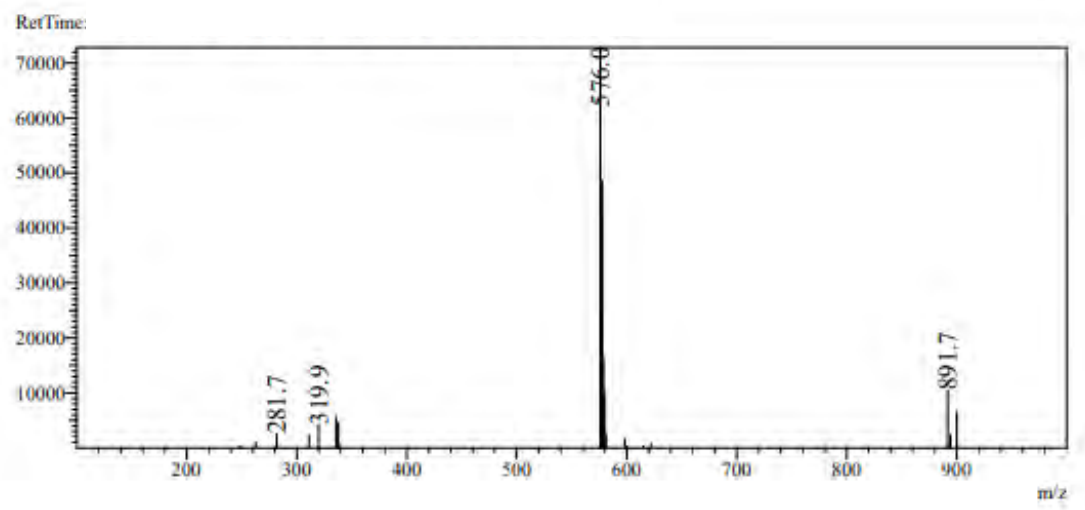

\section{Compound 6 1H NMR}




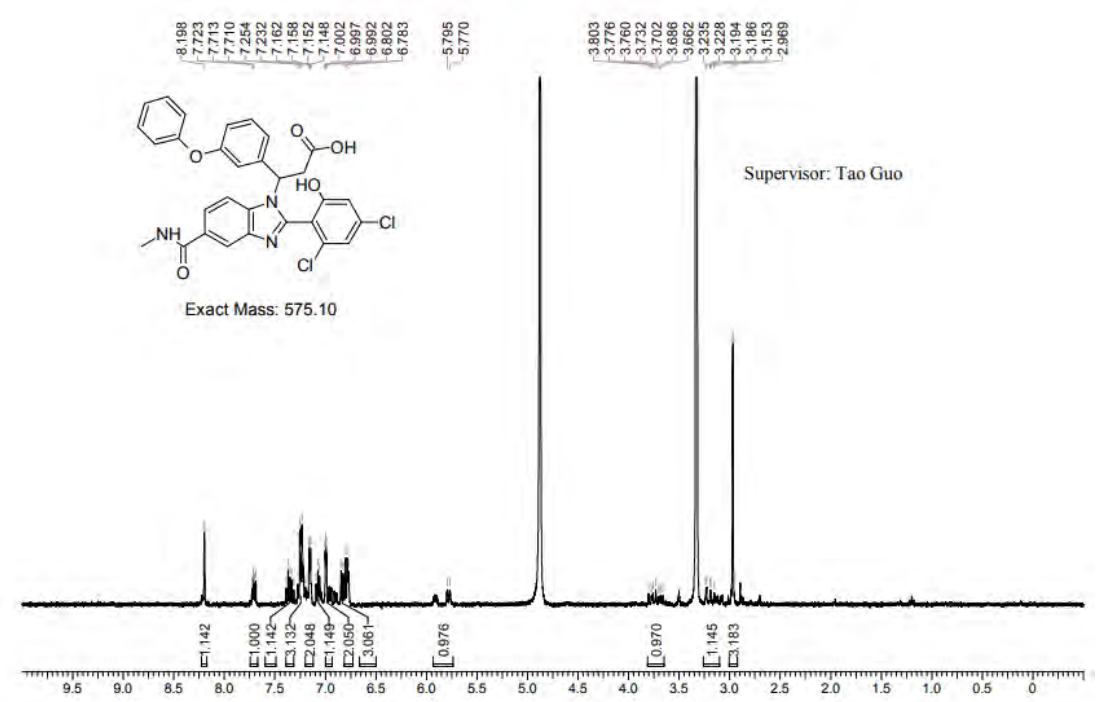

Compound 7:

\section{3-[2-(3-formyl-4-hydroxy-phenyl)-5-(methylcarbamoyl)benzimidazol-1-yl]-3-(3-} phenoxyphenyl)propanoic acid (Compound 7)
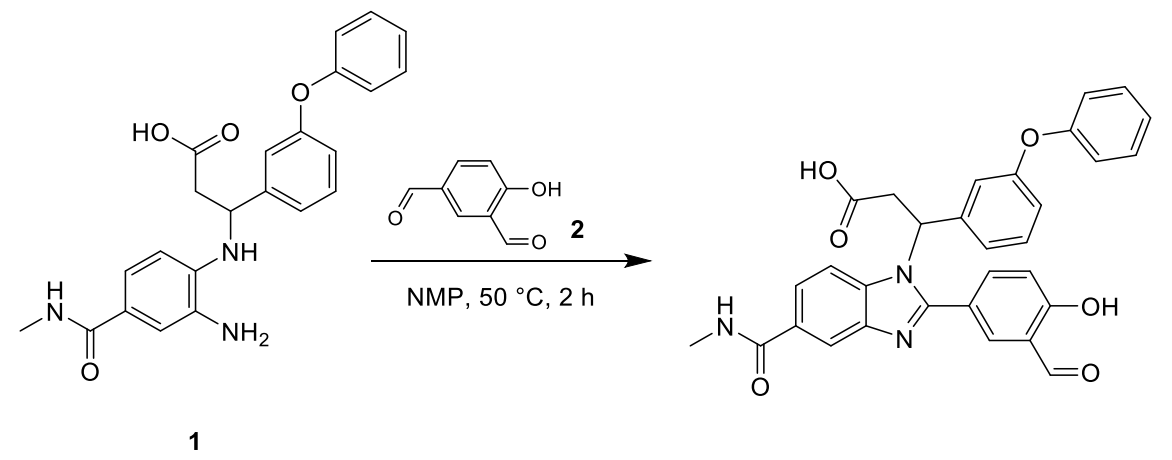

To a solution of 3-[2-amino-4-(methylcarbamoyl)anilino]-3-(3-phenoxyphenyl)propanoic acid (120.0 mg, $0.2000 \mathrm{mmol})$ in 1-methyl-2-pyrrolidinone $(3 \mathrm{~mL})$ was added 4-hydroxybenzene-1,3-dicarbaldehyde (33.24 mg, $0.2200 \mathrm{mmol}$ ) and oxone $(51.07 \mathrm{mg}, 0.3000 \mathrm{mmol})$. The mixture was stirred at $50{ }^{\circ} \mathrm{C}$ for $2 \mathrm{~h}$. LCMS (5-95AB/1.5min): RT = $0.881 \mathrm{~min},[\mathrm{M}+\mathrm{H}]^{+} 536.3$ showed $9.9 \%$ of DP. The crude product was purified by Prep-HPLC with the following conditions: Column, [Shim-pack C18 $150 \times 25 \mathrm{~mm}, 10 \mu \mathrm{m}$ ]; mobile phase: [ACN] and [water] (conditions: water(0.225\%FA)-ACN); Detector, UV $254 \mathrm{~nm}$. RT:[10 min]. The crude was further purified with GNEWX-1112-311 and purified by Prep-HPLC with the following conditions: Column, [Phenomenex Synergi C18 $150 \times 25 \mathrm{~mm}, 10 \mu \mathrm{m}$ ]; mobile phase: [ACN] and [water] (conditions: water $(0.2 \%$ FA)-ACN); Detector, UV 254 nm. RT: [11 min]. 3-[2-(3-formyl-4-hydroxy-phenyl)-5(methylcarbamoyl)benzimidazol-1-yl]-3-(3-phenoxyphenyl)propanoic acid (7.8mg,0.0141mmol, 7\% yield) 
was given as light yellow solid, confirmed by LCMS (5-95AB/1.5min): RT = $0.697 \mathrm{~min},[\mathrm{M}+\mathrm{H}]^{+} 536.1$ showed $97 \%$ of DP and 2D NMR.

1H NMR (400 MHz, DMSO-d6) $\delta=10.33(\mathrm{~s}, 1 \mathrm{H}), 8.43-8.38(\mathrm{~m}, 1 \mathrm{H}), 8.16-8.09(\mathrm{~m}, 1 \mathrm{H}), 7.91(\mathrm{~d}, \mathrm{~J}=2.1 \mathrm{~Hz}$, 1H), $7.81(b r d, J=9.0 ~ H z, 1 H), 7.72$ - $7.62(m, 1 H), 7.41(d, J=8.6 \mathrm{~Hz}, 1 \mathrm{H}), 7.37$ - $7.29(\mathrm{~m}, 3 \mathrm{H}), 7.19$ - 7.07 (m, 2H), 7.00 - $6.87(m, 4 H), 6.75(s, 1 H), 6.24-6.07(m, 1 H), 3.52(b r d, J=6.5 H z, 2 H), 2.81(d, J=4.4 \mathrm{~Hz}$, $3 \mathrm{H})$.

\section{Compound 7 LCMS}

$\mathrm{m} / \mathrm{L}$

Chrouatogram

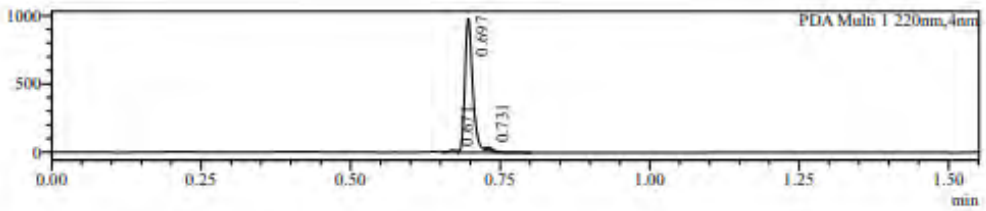

$\mathrm{mAl}$

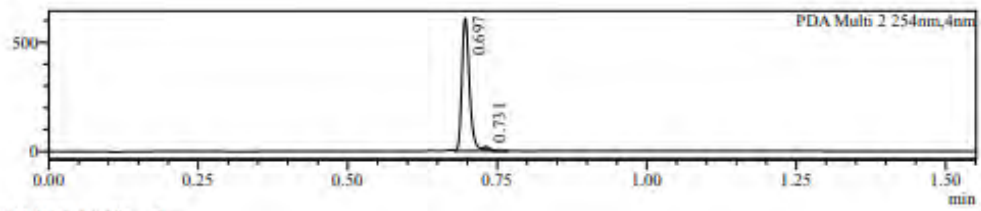

PDAMulti 1/220nm,4nm
PDAMulti $2 / 254 \mathrm{~nm}, 4 \mathrm{~nm}$

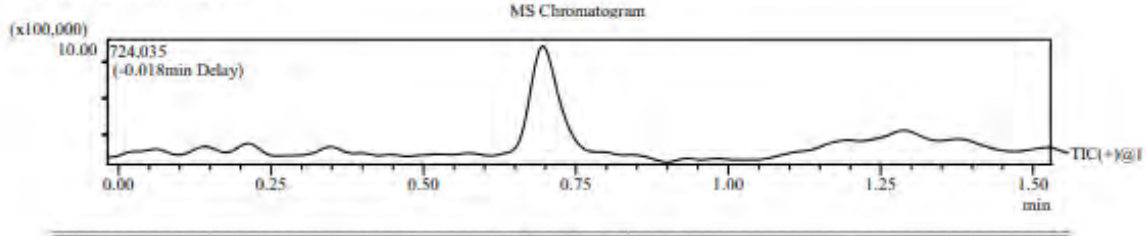

\begin{tabular}{|c|c|c|c|c|c|c|}
\hline & & \multicolumn{5}{|c|}{ Integration Result } \\
\hline & & & Pea & ik Table & & \\
\hline \multirow{4}{*}{$\begin{array}{r}\text { PDAChI } \\
\text { Peak } \# \\
1 \\
2 \\
3\end{array}$} & $\begin{array}{l}220 \mathrm{~nm} \\
\text { Ret. Time }\end{array}$ & Height & Height $\%$ & USP Width & & \\
\hline & 0.671 & 15256 & 1.507 & 0.027 & 12007 & 1.308 \\
\hline & 0.697 & 978572 & 96.667 & 0.025 & 894607 & 97.455 \\
\hline & 0.731 & 18489 & 1.826 & 0.019 & 11352 & 1.237 \\
\hline \multicolumn{7}{|c|}{ Peak Table } \\
\hline Peak\# & $\begin{array}{l}\text { 254nm } \\
\text { Ret. Time }\end{array}$ & Height & Height $\%$ & USP Width & Area & Area\% \\
\hline 1 & 0.697 & 608482 & 98.257 & 0.024 & 532300 & 98.739 \\
\hline 2 & 0.731 & 10794 & 1.743 & 0.020 & 6795 & 1.261 \\
\hline
\end{tabular}



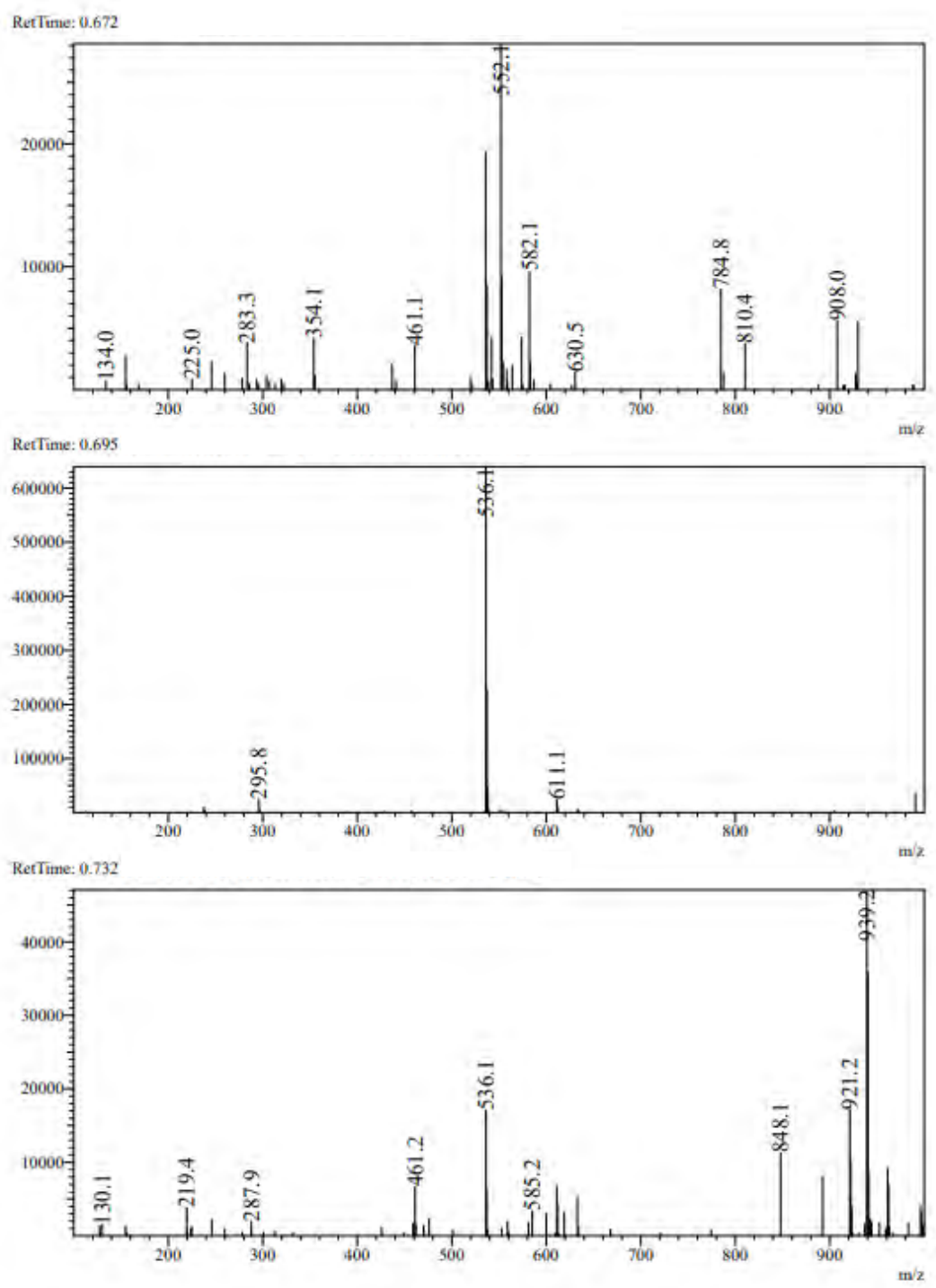

Compound 7 1H NMR 


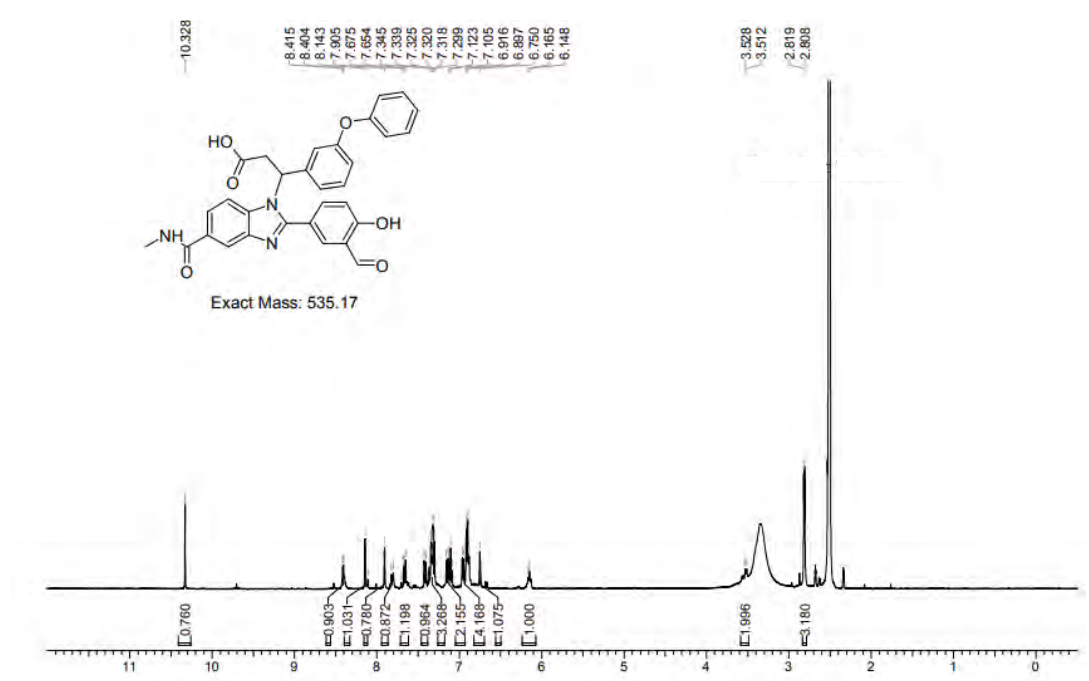

Compound 8:

3-[1-[2-carboxy-1-(4-methoxyphenyl)ethyl]-5-(methylcarbamoyl)benzimidazol-2-yl]-5-ethoxy-2hydroxy-benzoic acid (Compound 8)<smiles>CNC(=O)c1ccc(NC(CC(=O)O)c2ccc(OC)cc2)c(N)c1</smiles><smiles>CCOc1cc(OCC)cc(C(CC(=O)O)n2c(-c3cc(OCC)cc(C(=O)O)c3O)nc3cc(C(=O)NC)ccc32)c1</smiles>

To a solution of 3-[2-amino-4-(methylcarbamoyl)anilino]-3-(4-methoxyphenyl)propanoic acid (80.0 mg, $0.1900 \mathrm{mmol})$ in $\mathrm{N}, \mathrm{N}$-dimethylformamide $(1 \mathrm{~mL})$ was added 5-ethoxy-3-formyl-2-hydroxy-benzoic acid (43.09 $\mathrm{mg}, 0.2100 \mathrm{mmol})$. The mixture was stirred at $60^{\circ} \mathrm{C}$ for $12 \mathrm{~h}$. LCMS (5-95AB/1.5min): RT = 0.776 min, $[\mathrm{M}+\mathrm{H}]^{+} 534.3$ showed $63 \%$ of DP. The crude product was purified by Prep-HPLC with the following conditions: Column, [Phenomenex Synergi C18 $150 \times 25 \mathrm{~mm}, 10 \mu \mathrm{m}$ ]; mobile phase: [ACN] and [water] (conditions: water(0.225\%FA)-ACN); Detector, UV 254 nm. RT:[10 min]. 3-[1-[2-carboxy-1-(4methoxyphenyl)ethyl]-5-(methylcarbamoyl)benzimidazol-2-yl]-5-ethoxy-2-hydroxy-benzoic acid (35.3 $\mathrm{mg}, 0.0662 \mathrm{mmol}, 35.5 \%$ yield) was given as light yellow solid, confirmed by LCMS (5-95AB/1.5min): RT $=0.776 \mathrm{~min},[\mathrm{M}+\mathrm{H}]^{+} 534.2$ showed $100 \%$ of DP. 
$1 \mathrm{H} \mathrm{NMR}(400 \mathrm{MHz}$, methanol-d4) $\delta=8.19(\mathrm{~d}, \mathrm{~J}=1.2 \mathrm{~Hz}, 1 \mathrm{H}), 7.73-7.66(\mathrm{~m}, 2 \mathrm{H}), 7.37-7.31(\mathrm{~m}, 2 \mathrm{H}), 7.24$ $(\mathrm{d}, \mathrm{J}=8.7 \mathrm{~Hz}, 2 \mathrm{H}), 6.88(\mathrm{~d}, \mathrm{~J}=8.8 \mathrm{~Hz}, 2 \mathrm{H}), 6.02(\mathrm{t}, \mathrm{J}=7.4 \mathrm{~Hz}, 1 \mathrm{H}), 4.08(\mathrm{~d}, \mathrm{~J}=7.0 \mathrm{~Hz}, 2 \mathrm{H}), 3.78(\mathrm{~s}, 3 \mathrm{H}), 3.69$ (dd, J = 8.6, $16.0 \mathrm{~Hz}, 1 \mathrm{H}), 3.38(\mathrm{br} \mathrm{d}, \mathrm{J}=6.5 \mathrm{~Hz}, 1 \mathrm{H}), 2.96(\mathrm{~s}, 3 \mathrm{H}), 1.42(\mathrm{t}, \mathrm{J}=7.0 \mathrm{~Hz}, 3 \mathrm{H})$.

\section{Compound 8 LCMS}

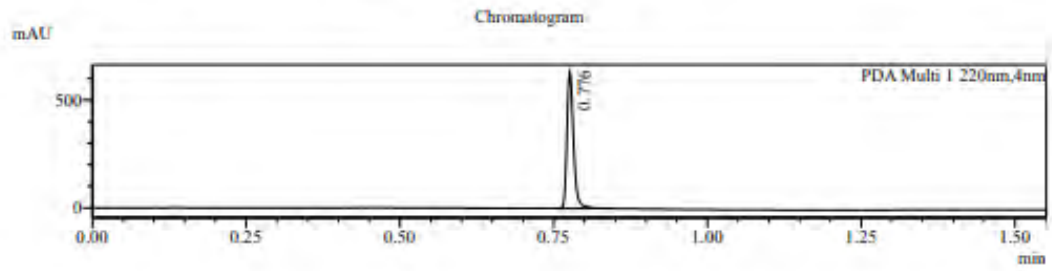

$\mathrm{mAC}$

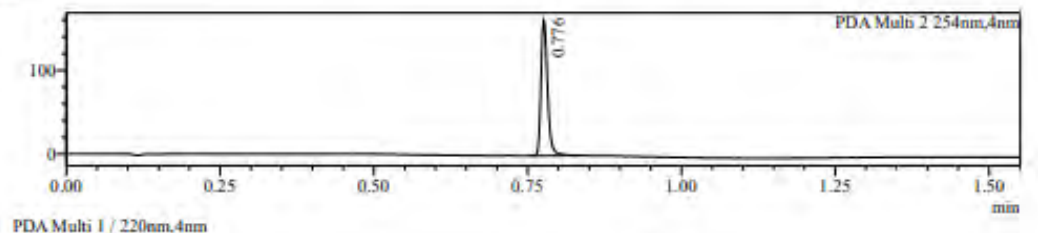

2 PDA Multi 2/254nm,4nm
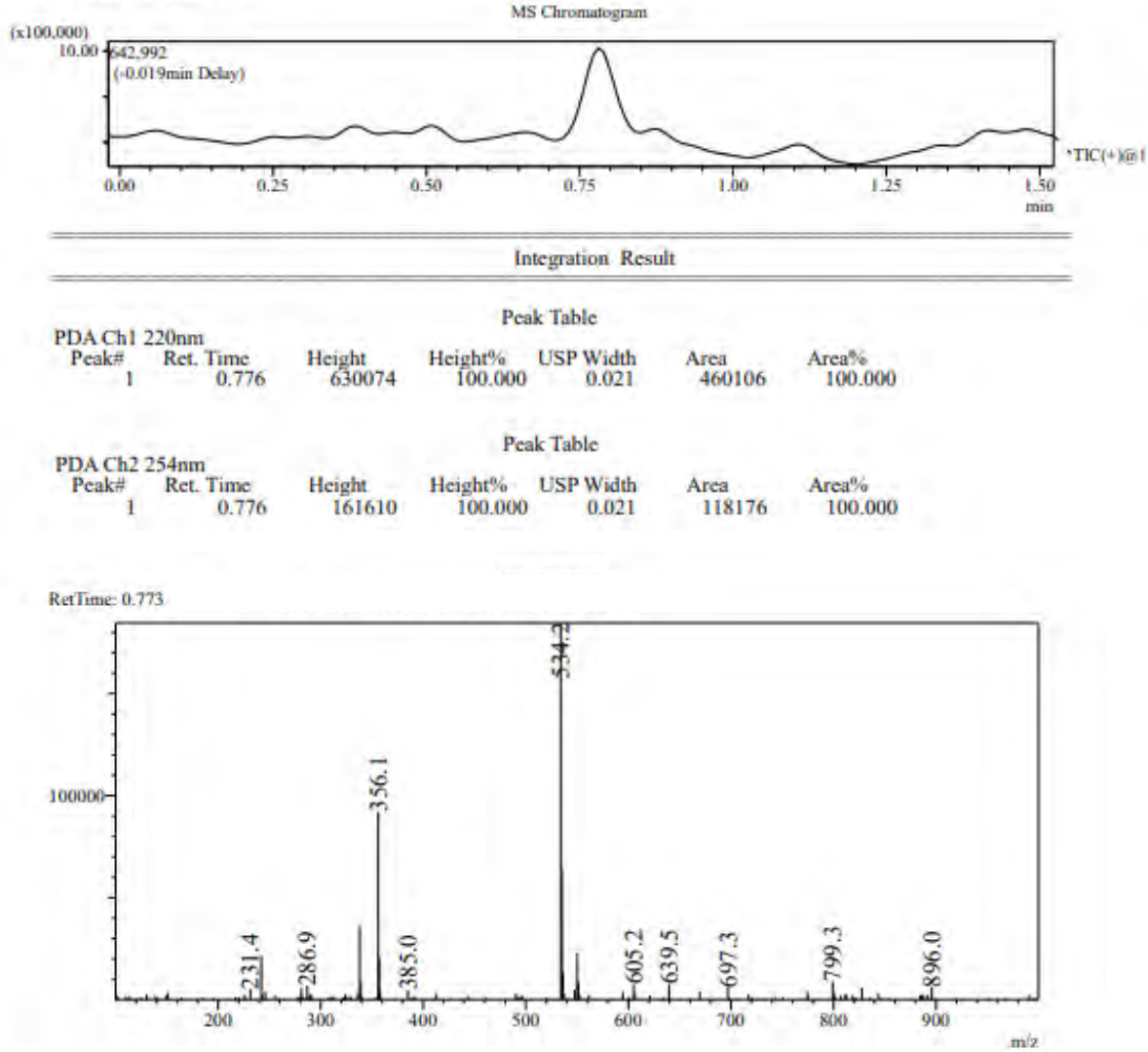

\section{Compound 8 1H NMR}




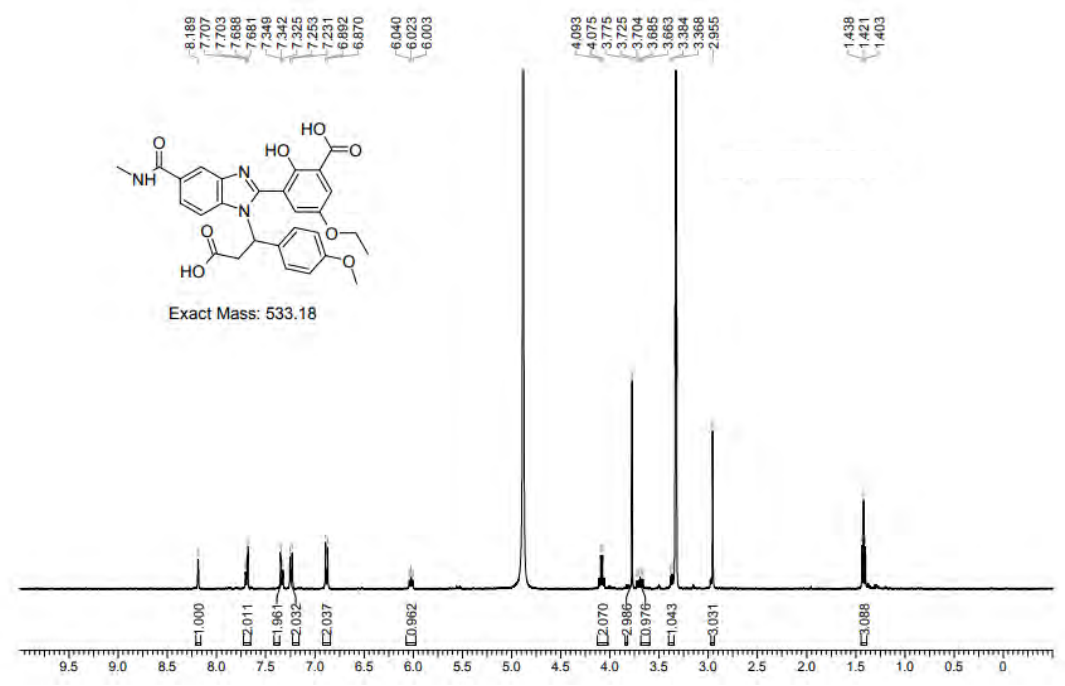

Compound 9:

5-ethoxy-3-formyl-2-hydroxybenzoic acid (4)<smiles>CCOc1ccc(O)c(C(=O)O)c1</smiles>

2

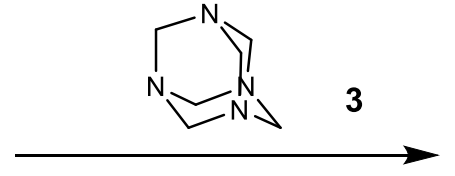

TFA, $90{ }^{\circ} \mathrm{C}, 12 \mathrm{~h}$<smiles>CCOc1cc(C=O)c(O)c(C(=O)O)c1</smiles>

4

To a solution of 5-ethoxy-2-hydroxy-benzoic acid (1.77 g, $9.72 \mathrm{mmol}$ ) in 2,2,2-trifluoroacetic acid (20 mL) was added hexamethylenetetramine $(1634.49 \mathrm{mg}, 11.66 \mathrm{mmol})$. The mixture was stirred at $90^{\circ} \mathrm{C}$ for $12 \mathrm{~h}$ under $\mathrm{N}_{2}$. The TLC (100\% ethyl acetate, $\left.\mathrm{Rf}=0.2\right)$ indicated the main spot was formed. The mixture was concentrated and added $4 \mathrm{M} \mathrm{HCl}(20 \mathrm{~mL})$. After stirred at $20^{\circ} \mathrm{C}$ for $10 \mathrm{~min}$, the mixture was extracted with DCM $(50 \mathrm{~mL} \times 3)$ and the organic layers were combined. 5-ethoxy-3-formyl-2-hydroxy-benzoic acid (1.25 g, $5.5904 \mathrm{mmol}, 57.5 \%$ yield) was given as light yellow solid. LCMS (5-95AB/1.5min): RT =0.669 min, $[\mathrm{M}+\mathrm{H}]^{+} 211.1$ showed $94 \%$ of DP.

1H NMR (400 MHz, DMSO-d6) $\delta=10.36(s, 1 H), 7.61(d, J=3.3 \mathrm{~Hz}, 1 \mathrm{H}), 7.43(\mathrm{~d}, \mathrm{~J}=3.3 \mathrm{~Hz}, 1 \mathrm{H}), 4.05(\mathrm{q}, \mathrm{J}$ $=6.9 \mathrm{~Hz}, 2 \mathrm{H}), 1.32(\mathrm{t}, \mathrm{J}=6.9 \mathrm{~Hz}, 3 \mathrm{H})$

3-[1-[2-carboxy-1-(4-fluorophenyl)ethyl]-5-(methylcarbamoyl)benzimidazol-2-yl]-5-ethoxy-2-hydroxybenzoic acid (Compound 9) 

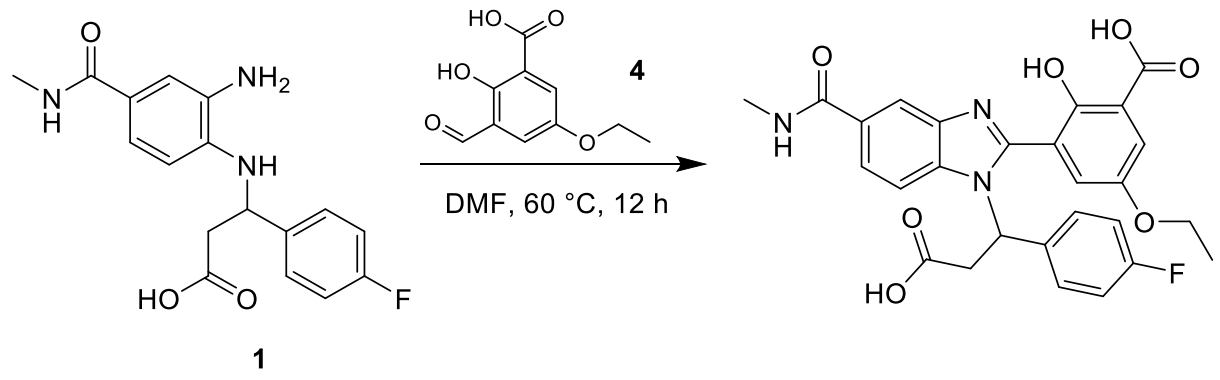

To a solution of 3-[2-amino-4-(methylcarbamoyl)anilino]-3-(4-fluorophenyl)propanoic acid (80.0 mg, $0.2300 \mathrm{mmol}$ ) in $\mathrm{N}, \mathrm{N}$-Dimethylformamide $(1 \mathrm{~mL})$ was added 5-ethoxy-3-formyl-2-hydroxy-benzoic acid (52.47 mg, $0.2500 \mathrm{mmol})$. The mixture was stirred at $60^{\circ} \mathrm{C}$ for $12 \mathrm{~h}$. LCMS (5-95AB/1.5min): RT = 0.786 min, $[\mathrm{M}+\mathrm{H}]^{+} 522.2$ showed $76 \%$ of DP. The crude product was purified by Prep-HPLC with the following conditions: Column, [Phenomenex Synergi C18 $150 \times 25 \mathrm{~mm}, 10 \mu \mathrm{m}$ ]; mobile phase: [ACN] and [water] (conditions: water(0.225\%FA)-ACN); Detector, UV $254 \mathrm{~nm} . \mathrm{RT}:[10 \mathrm{~min}]$. 3-[1-[2-carboxy-1-(4fluorophenyl)ethyl]-5-(methylcarbamoyl)benzimidazol-2-yl]-5-ethoxy-2-hydroxy-benzoic acid (41.7 mg, $0.0800 \mathrm{mmol}, 35.2 \%$ yield) was given as light yellow solid, confirmed by LCMS (5-95AB/1.5min): RT = 0.620 min, $[\mathrm{M}+\mathrm{H}]^{+} 522.1$ showed $100 \%$ of DP.

1H NMR (400 MHz, methanol-d4) $\delta=8.20(\mathrm{~s}, 1 \mathrm{H}), 7.74-7.66(\mathrm{~m}, 2 \mathrm{H}), 7.43-7.29(\mathrm{~m}, 4 \mathrm{H}), 7.08(\mathrm{t}, \mathrm{J}=8.7$ $\mathrm{Hz}, 2 \mathrm{H}), 6.04(\mathrm{t}, \mathrm{J}=7.4 \mathrm{~Hz}, 1 \mathrm{H}), 4.09(\mathrm{q}, \mathrm{J}=7.1 \mathrm{~Hz}, 2 \mathrm{H}), 3.72(\mathrm{dd}, \mathrm{J}=8.1,16.3 \mathrm{~Hz}, 1 \mathrm{H}), 3.39$ (dd, J = 6.6, 16.3 $\mathrm{Hz}, 1 \mathrm{H}), 2.96(\mathrm{~s}, 3 \mathrm{H}), 1.42(\mathrm{t}, \mathrm{J}=7.0 \mathrm{~Hz}, 3 \mathrm{H})$

\section{Compound 9 LCMS}



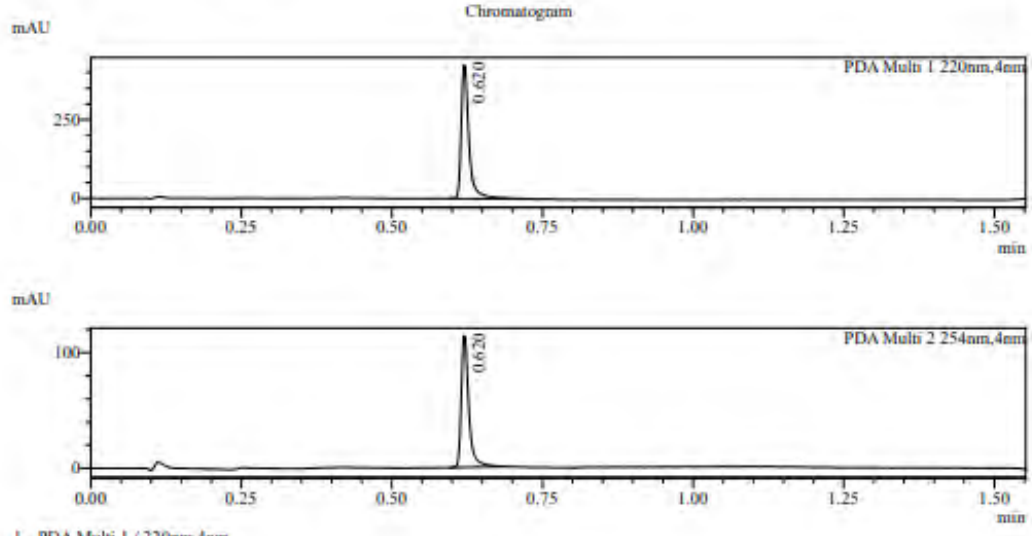

I PDN Matifl 220nm, na

MS Chromatoeram

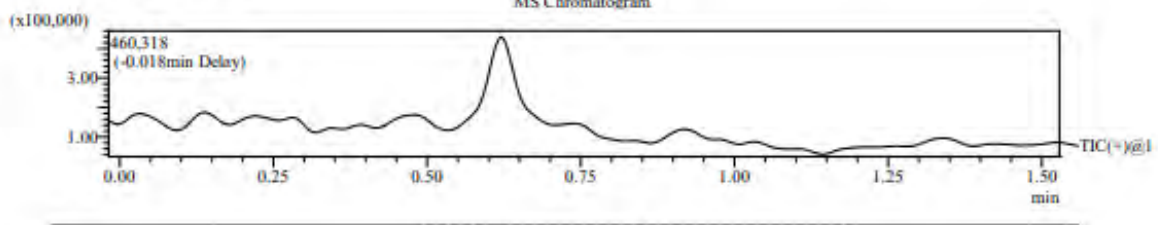

\begin{tabular}{ll}
\hline \hline & Integration Result \\
\hline \hline PDAChl 220nm & Peak Table
\end{tabular}

PDAChl 220nm
Peak\# Ret. Time Height Height\% USP Width Area Area\%

$\begin{array}{llllllll}1 & 0.620 & 425021 & 100.000 & 0.023 & 376957 & 100.00\end{array}$
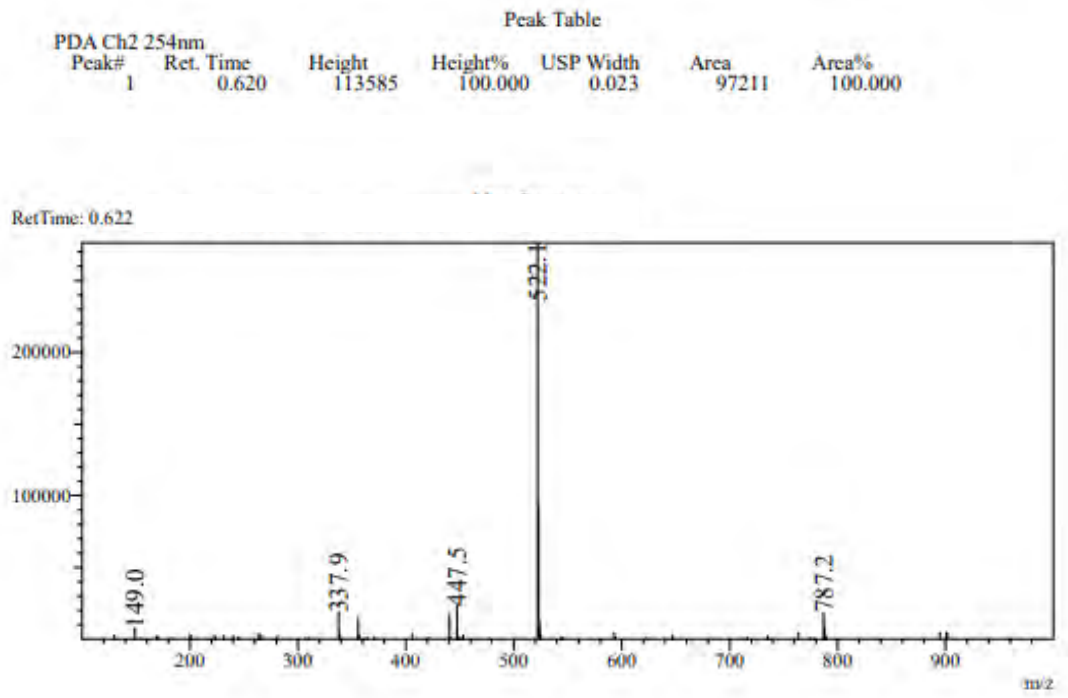

\section{Compound 9 1H NMR}




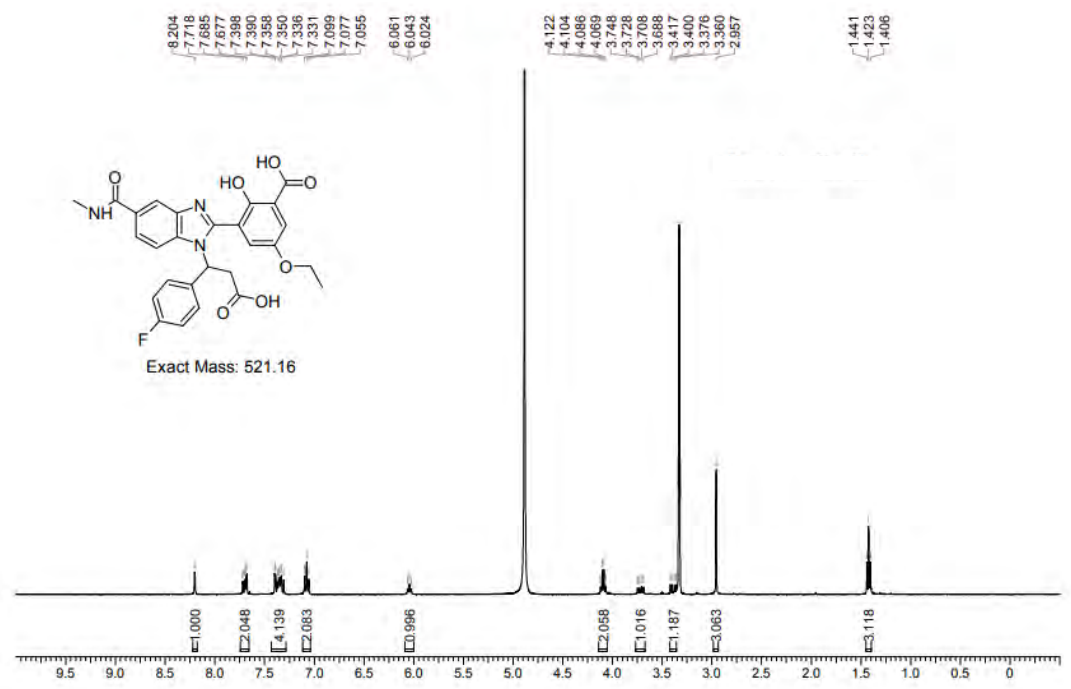

